\title{
Coalitional Game Theoretic Approach for Secondary Spectrum Access in Cooperative Cognitive Radio Networks
}

\author{
Dapeng Li, Youyun Xu, Member, IEEE, Xinbing Wang, Member, IEEE, and Mohsen Guizani, Fellow, IEEE
}

\begin{abstract}
In this paper, we exploit a novel setting for Cognitive Radio (CR) networks to enable multiple operators to involve secondary users (SUs) as cooperative relays for their primary users. In return, SUs get an opportunity to access spare channels for their own data transmission. Initially, we assume that the CR network supports payment transfer. Then, we formulate the system as a transferable utility coalitional game. We show that there is an operating point that maximizes the sum utility over all operators and SUs while providing each player a share such that no subset of operators and SUs has an incentive to break away from the grand coalition. Such operating points exist when the solution set of the game, the core, is nonempty. Subsequently, we examine an interesting scenario where there is no payment mechanism in the network. This scenario can be investigated by using a nontransferable utility coalitional game model. We show that there exists a joint action to make the core nonempty. A general method with exponential computational complexity to get such a joint action is discussed. Then, we relate the core of this game to a competitive equilibrium of an exchange economy setting under special situations. As a result, several available efficient centralized or distributed algorithms in economics can be employed to compute a member in the core. In a nutshell, this paper constitutes the design of new coalition based dynamics that could be used in future CR networks.
\end{abstract}

Index Terms-Cognitive radio networks, cooperative diversity transmission, coalitional game, core, exchange economy, competitive equilibrium.

\section{INTRODUCTION}

$\mathbf{I}$ $\mathrm{N}$ the past few years, we have witnessed a significant growth in commercial wireless services. In a recent FCC report about significant unbalance of spectrum usage, it was stated that some frequency bands are largely unoccupied most of the time; whereas others are only partially occupied; the remaining frequency bands are heavily used [1]. Cognitive radio or secondary spectrum access has been viewed as a novel

Manuscript received February 12, 2010; revised July 2, 2010, October 10, 2010, and December 1, 2010; accepted December 16, 2010. The associate editor coordinating the review of this paper and approving it for publication was M. Buehrer.

D. Li, Y. Xu, and X. Wang are with the Department of Electronic Engineering, Shanghai Jiao Tong University, Shanghai, China (e-mail: \{lidapeng, xwang8\}@sjtu.edu.cn, yyxu@vip.sina.com).

M. Guizani is with the Information Science Department, Kuwait University, Kuwait (e-mail: mguizani@ieee.org).

$\mathrm{D}$. $\mathrm{Li}$ is also affiliated with the College of Telecommunications and Information Engineering, Nanjing University of Posts and Telecommunications, Nanjing, China. Y. Xu is also also affiliated with the Nanjing Institute of Communication Engineering, PLA University of Science and Technology, Nanjing, China.

Digital Object Identifier 10.1109/TWC.2011.011111.100216 approach for improving the spectrum utilization by allowing unlicensed SUs to access licensed spectra.

Facing the challenges brought by the demand in spectrum availability, cooperative diversity transmissions have also been proposed as important techniques to take advantage of the broadcast nature of wireless medium and provide improved spectrum efficiency. Motivated by cooperative communications, we know that if SUs serve as cooperative relays for primary transmissions, the transmissions' rates can be dramatically increased by exploiting cooperative diversity. This will generate higher revenue for OPs from subscribers/primary users (PUs). In return, SUs are allowed to access the unoccupied bands of OPs. We call such a scenario as Cooperative Secondary Spectrum Access (CSSA) in which both the primary system and the secondary system can increase their own interest and a win-win situation can be achieved.

In regard to exploiting the cooperation between a primary system and a secondary system in CR networks, there are only two existing research articles (to the best of our knowledge) which considered the scenario where one PU leases its spectrum to SUs for a fraction of time and in exchange, it gets the revenue or the cooperative transmission power from SUs [2-3]. The authors investigated their models using Stackerberg games. In such systems, time is exactly a kind of resource and should be well allocated, i.e., the fraction of time allocated to the transmissions of SUs will be determined by the negotiation among game players. The major difference between [2-3] and our work lies on that our model captures the interaction between multiple OPs and multiple SUs.

The success of CSSA is however contingent upon developing a rational basis for the joint cooperative actions/strategies of OPs and SUs. On one hand, multiple SUs compete with each other to access the spare bands of multiple OPs. On the other hand, multiple OPs compete with each other to get the cooperative transmissions from multiple SUs. Hence, a gametheoretic analysis is needed for such situations. Coalitional games proved to be powerful tools for designing fair, robust, practical and efficient cooperation strategies in different disciplines such as economics or political science. The standard coalitional game techniques [17] defined the fundamental components of the games. However, many coalitional games are inherently difficult to solve. In this paper, we apply coalitional game and economic theories to analyze the network behavior in CCSA. Using Transferable Utility coalitional (TU) game theory, we first develop the framework assuming that 
OPs and SUs can share the aggregate payoff arbitrarily. They form coalitions to achieve their own best payoff in terms of performance and price. We show that the core of the TU game is nonempty and a payoff allocation in the core can be obtained by a dual solution of a convex optimization problem. As a result, the grand coalition is a stable and optimal solution of the game.

Undoubtedly, introducing trading/payment mechanisms into CR networks will involve extra implementation cost. An interesting direction for CSSA is to study the scenario where OPs and SUs cannot share the aggregate payoff in any manner they wish to. Such a situation leads to a Non-Transferable Utility coalitional (NTU) game model. Furthermore, we show that the core of the game is non-empty. We discuss a general method with exponential computational complexity to get an element in the core. However, under special situations, we can construct a pure "exchange market" setting that is related to the resulting game. We show that in this setting, a Competitive Equilibrium (CE) exists. Consequently, the allocation strategy of goods in the market setting given by the $\mathrm{CE}$ can be translated into a joint action among OPs and SUs with the corresponding payoffs in the core.

In summary, we make the following contributions in this paper:

- This work proposes a new spectrum sharing model for CR networks, which can enable the cooperation between multiple OPs and multiple SUs. The model is investigated with and without a payment mechanism.

- The system with a payment mechanism is formulated as a TU game where OPs and SUs can pay charges to each other to motivate cooperation. We obtain an element in the core of the game using a dual solution of a convex problem. Indeed, the analysis identifies the pricing issue for stable and optimal cooperative strategies of OPs and SUs.

- Furthermore, we examine cooperation without payment transfer using an NTU game. We prove that the core of such a game is nonempty, and relate the game to an exchange economy setting. Under special conditions, we show that a $\mathrm{CE}$ of the economy setting corresponds to a payoff allocation in the core of the NTU game. Then, the method to compute an element in the core is presented.

- Based on the analytical results, the implementation of CSSA is discussed in detail for each case. Numerical analysis illustrates how OPs and SUs cooperate to increase their own interest. In addition, the analysis also shows different solutions between the TU game and the NTU game.

The remainder of the paper is organized as follows: Section II reviews the related research work. Section III describes the system model. In Section IV, we formulate the TU game and obtain a solution of the game using a duality technique of convex optimization. Section V investigates the situation without payment transfer. An NTU game model is proposed in this section. Section VI focuses on the computation of the solution of the NTU game. We discuss the implementation of CSSA in Section VII, and numerical results are presented in Section VIII. Finally, we conclude the paper in Section IX.

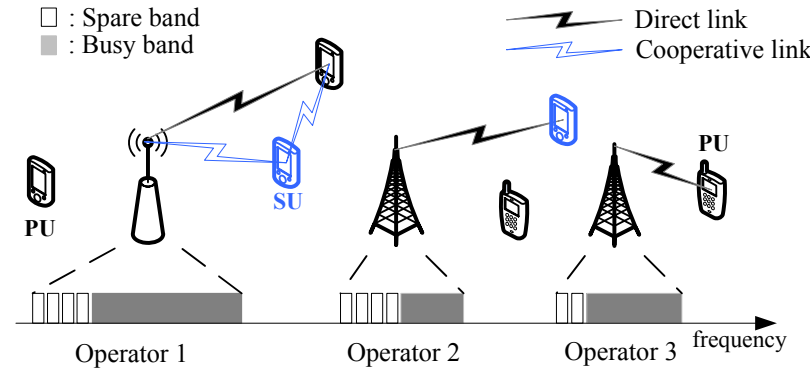

Fig. 1. System model.

\section{RELATED RESEARCH WORK}

Many previous research work in this area addressed issues of pricing in dynamic spectrum access environments. These included transmit power auctions which were proposed by Huang et al. [4]. In [4], bidders use the same spectrum band but bid transmit power to minimize the interference. Later, several spectrum trading models were presented by Niyato and Hossain [5]. Kasbekar et al. [6] formulated a spectrum auction framework to maximize the auctioneer's revenue or the social welfare. Niyato et al. [7] used a non-cooperative game to analyze the dynamic spectrum trading between multiseller and multi-buyer in CR networks. Yu et al. [29] proposed a pricing based scheme for uplink power control in CR networks. Wang et al. studied an auction game for spectrum sharing [30]. Li et al. explored an underground coal mine monitoring model in wireless sensor networks [31]. However, all of these attempts did not employ cooperative transmissions.

The survey presented in [8] classified coalitional games into three categories: canonical coalitional games, coalition formation games and coalitional graph games. Such a new classification is intended to provide an application-oriented approach to coalitional games. The game models used in this paper belong to the first class which constitutes one of the most classical types of games. More details on the state-ofthe-art research contributions of different classes of coalitional games were discussed in [8]. Canonical coalitional games have been used recently for modeling the cooperation among nodes in multi-hop networks [9]. Cooperation between single antenna receivers and transmitters in an interference channel has been studied by Mathur et al. [10]. Cooperation among different operators to serve each other's subscribers in wireless networks using canonical coalitional game theory has been investigated by Aram et al. [11], [12]. The specific coalitional games we consider and the analytical results we obtain in this paper, significantly differ from those of the above papers.

\section{SYSTEM ARChiteCtURE}

In this section, we present an overview of the system architecture of CSSA. As shown in Fig. 1, there is a set of N service operators and a set of K SUs in the same region. OP $i, i \in \mathrm{N}$ has a licensed spectrum which is unbalanced occupied. For instance, there are overloaded spectrum demands in OFDMA networks. However, there may be some spare bands in other mobile networks, e.g., GSM networks. OPs can improve the throughput in the overloaded spectrum by involving SUs as 
TABLE I

MAIN NOTATIONS

\begin{tabular}{|c|c|}
\hline & Basic Notations \\
\hline \multirow[t]{2}{*}{$\begin{array}{l}\beta_{m, k} \\
\alpha_{j, k}\end{array}$} & $\begin{array}{l}\text { The fraction of time SU } k \text { uses to assist PU } m \text {. } \\
\text { The fraction of time SU } k \text { gets to access band } j \text {. }\end{array}$ \\
\hline & Notations in Sections III, IV and V. \\
\hline \multirow[t]{2}{*}{$\begin{array}{l}x_{i} \\
\mathbf{x}\end{array}$} & 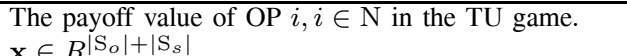 \\
\hline & A payoff allocation for coalition $\mathrm{S}$ in the $\mathrm{TU}$ game. \\
\hline \multirow[t]{2}{*}{$f_{i}$} & The payoff value of OP $i, i \in \mathrm{N}$ in the NTU game. \\
\hline & Notations in Section VI. \\
\hline $\begin{array}{l}j^{\prime} \\
k^{\prime}\end{array}$ & $\begin{array}{l}\text { The rank number of band } j \text { in } \mathrm{N}_{b} \text {. } \\
\text { The rank number of SU } k \text { in } K \text {. }\end{array}$ \\
\hline$x_{i}$ & $x_{i} \in R_{+}^{\left(\left|\mathrm{N}_{b}\right|+|\mathrm{K}|\right)}$. A resource vector of OP $i, i \in \mathrm{N}$. \\
\hline $\mathbf{x}$ & $\mathbf{x} \in R_{+}^{(|\mathrm{N}|+|\mathrm{K}|)\left(\left|\mathrm{N}_{b}\right|+|\mathrm{K}|\right)}$ \\
\hline & A resource allocation of the economy setting. \\
\hline
\end{tabular}

cooperative relays. In return, SUs are allowed to access the spare bands.

Denote the set of the spare bands of OP $i$ as $\mathrm{B}_{i}$. Let $\mathrm{M}_{i}$ represent the set of PUs in the overloaded spectrum of OP $i$. A spare band owned by $\mathrm{OP} i$ can serve $\mathrm{SU} k, k \in \mathrm{K}$ only if both are in a coalition ${ }^{1}$. Similarly, an SU can assist the transmission of a PU only when the SU and the OP associated with this PU are in a coalition. Before we describe the actions of OPs and SUs, we first give the definition of a coalition in such a system.

Definition 1: A coalition $S$ is a subset of players (e.g., operators and SUs) that cooperate. For a coalition S, let $\mathrm{S}_{o}$ and $\mathrm{S}_{s}$ represent the set of OPs and SUs in S, respectively. $\mathrm{S}_{b}$ denotes the set of the spare bands of the OPs in $\mathrm{S} . \mathrm{S}_{p}$ represents the set of the PUs associated with the OPs in S. Denote $\mathrm{N} \cup \mathrm{K}$ as the grand coalition consisting of all OPs and SUs. Accordingly, let $\mathrm{N}_{p}$ be the set of all PUs and $\mathrm{N}_{b}$ be the set of all spare bands. Throughout the paper, we use symbol |.| to mean the cardinality of a set.

\section{A. Cooperative secondary spectrum access}

We assume that no PUs have access to the same frequency band. The bandwidth of the channel assigned to each PU is assumed to be "one". In this paper, we assume that SUs/relays and PUs use a fixed transmission power $P$. Let $\delta_{v}^{2}$ represent the noise level at each receiver. Without the cooperation from relays, the direct transmission data rate of PU $m$ is $I_{m}=\log \left(1+\frac{P}{\delta_{v}^{2}}\left|g_{m}\right|\right)$, where $g_{m}$ is the channel gain between PU $m$ and the access point of its OP. Each OP in coalition $\mathrm{S}$ selects several SUs from $\mathrm{S}_{s}$ to relay signals for its PUs. In the following parts of the paper, we employ the nonorthogonal amplify-and-forward (NAF) cooperation protocol [13], [15] for the cooperative transmissions. Other protocols can be considered in a similar way. Assume that the SU(s) cooperating with PU $m$ use(s) the same spectrum assigned to this PU. Let $\rho_{k, m}$ denote the amplify factor of SU $k$ for PU $m$. According to [13], [14], $\rho_{k, m}=\sqrt{\frac{P}{P\left|h_{i, k}\right|^{2}+\delta_{k}^{2}}}$ where $h_{i, k}$ is the channel gain between $\mathrm{OP} i$ and relay/SU $k . \delta_{k}^{2}$ is the

\footnotetext{
${ }^{1}$ A coalitional model is distinguished from a noncooperative model mainly by its focus on what groups of players can achieve rather than what individual players can do.
}

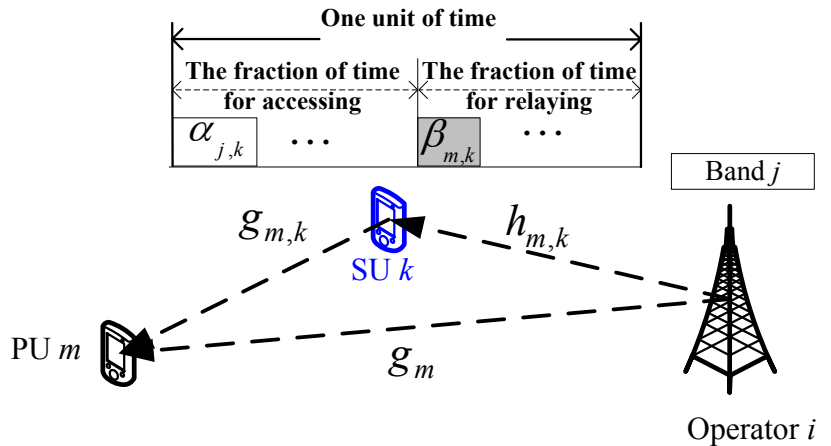

Fig. 2. Cooperative Secondary Spectrum Access.

noise power at $\mathrm{SU} k$. We denote the data rate increase of $\mathrm{PU}$ $m$ with the cooperation from relay/SU $k$ as $I_{k, m}$.

Suppose that one SU assists the transmission of at most one PU at a given time. In one unit of time ${ }^{2}$, let $\beta_{m, k} \in[0,1]$ denote the fraction of time $\mathrm{SU} k, k \in \mathrm{S}_{s}$ uses to assist the transmission of PU $m, m \in \mathrm{S}_{p}$. Then, the data rate increase of PU $m$ in coalition $\mathrm{S}$ is $y_{m}^{\prime}=\sum_{k \in \mathrm{S}_{s}} \beta_{m, k} I_{k, m}$. Thus, the throughput increase of $\mathrm{OP} i$ in coalition $\mathrm{S}$ is:

$$
y_{i}=\sum_{m \in \mathrm{M}_{i}} y_{m}^{\prime}
$$

Each OP licenses certain frequency bands. Thus, a spare band is licensed by at most one OP. We assume that no two SUs in a vicinity are assigned to the same spare band at a given time. When $\mathrm{SU} k, k \in \mathrm{S}_{s}$ accesses band $j$, it receives a rate $R_{j, k}$ which depends on the location of SU $k$ and the channel gain of frequency band $j$. For coalition $\mathrm{S}$, let $\alpha_{j, k} \in[0,1]$ denote the fraction of time SU $k$ gets to access band $j, j \in \mathrm{S}_{b}$. Figure 2 presents an example for the CSSA. The total access rate achieved by SU $k$ in one unit of time is:

$$
r_{k}=\sum_{j \in \mathrm{S}_{b}} \alpha_{j, k} R_{j, k} .
$$

The fraction of the access time SU $k$ uses to access band $j$ is $w_{j}^{\prime}=\sum_{k \in \mathrm{S}_{s}} \alpha_{j, k}$. The expected cost of an OP depends on the total fraction of the access time it provides to SUs, which can be represented as:

$$
w_{i}=\sum_{j \in \mathrm{B}_{i}} w_{j}^{\prime} .
$$

The total energy consumption of SU $k$ serving as relays in coalition $\mathrm{S}$ is:

$$
e_{k}=\sum_{m \in \mathrm{S}_{p}} \frac{1}{2} \beta_{m, k} P,
$$

where $1 / 2$ is due to the half of transmission time of the cooperative protocol. The main notations of this paper are shown in Table I. Throughout the paper, we assume that each SU has a half-duplex constraint. At a given time, an SU either works in relay or access mode, but not both.

\footnotetext{
${ }^{2}$ We assume that OPs and SUs perform cooperation in one unit time, and the network topology and channel conditions do not change during the cooperation.
} 


\section{B. Utility functions and problem description}

Each PU pays its OP with a fixed charging policy (i.e., according to the QoS of the PU). Thus, with the increase of data rates of PUs, OP $i$ generates a throughput gain of $U_{i}^{+}\left(y_{i}\right)$ where $U_{i}^{+}($.$) is a concave increasing function and$ reflects the aggregate satisfaction of the PUs subscribed to OP $i$. For example, utility functions can be linear, i.e., $U_{i}^{+}\left(y_{i}\right)=$ $y_{i}$. OP $i$ incurs cost when SUs access its channels. We define the cost as a function $U_{i}^{-}($.$) of w_{i}$. Specifically, the secondary access cost of OP $i$ in coalition $\mathrm{S}$ is $U_{i}^{-}\left(w_{i}\right)$ where $U_{i}^{-}($. is an increasing convex function. Then, the utility evaluation of OP $i$ is $U_{i}^{+}\left(y_{i}\right)-U_{i}^{-}\left(w_{i}\right)$. The access rate gain of $\mathrm{SU}$ $k$ can be evaluated by a function $G_{k}^{+}($.$) where G_{k}^{+}($.$) is an$ increasing concave function. SUs could be mobile users and may be sensitive to energy conditions. Thus, we define the total energy cost of SU $k$ serving as relays in a coalition as $G_{k}^{-}\left(e_{k}\right)$ where $G_{k}^{-}($.$) is an increasing convex function. Hence,$ the utility evaluation of SU $k$ is the access rate gain reduced by the energy cost, $G_{k}^{+}\left(r_{k}\right)-G_{k}^{-}\left(e_{k}\right)$. A similar utility function has been employed in [2] where the energy cost function of an SU is a linear function.

In spectrum trading, pricing is a key issue of interest to primary service providers (i.e., spectrum owners) as well as to SUs. In a CR network, pricing model for spectrum sharing depends on the objective of spectrum trading, and therefore, the behaviors of spectrum sellers and spectrum buyers [5]. In some spectrum trading models (e.g.,[6-9]), the utility function of an SU was often defined to be its achievable rate in equivalent revenue format minus the payment it makes for the primary user or the spectrum owner. For CSSA, we also presume that the utility evaluation of each OP or SU is in equivalent revenue format. Then, when the system supports a trading mechanism, OPs and SUs will form cooperative groups/coalitions to achieve their own best payoff in terms of utility evaluations and the payment they obtain or pay. Hence, the utility of SU $k$ in a coalition is:

$$
G_{k}^{+}\left(r_{k}\right)-G_{k}^{-}\left(e_{k}\right) \pm p_{k}
$$

where $\pm p_{k}$ represents the payment SU $k$ obtains or pays in the coalition, and can be transferred between OPs and SUs. Note that the utility evaluation of each player defined here is not transferable, and is exactly determined by the cooperative strategies of all players in the coalition. For the NTU model, $\pm p_{k}$ always equals zero. Similarly, the utility of OP $i$ in a coalition is:

$$
U_{i}^{+}\left(y_{i}\right)-U_{i}^{-}\left(w_{i}\right) \pm p_{i}
$$

Every commercial OP or SU is a selfish entity that seeks to maximize the individual payoff. Therefore, OPs and SUs will form a coalition only when this increases their own payoff. However, implementing coalition based dynamics faces several challenges such as adequate modeling, efficiency, complexity and fairness. Using tools from coalitional game and economic theories, this paper is intended to develop techniques to study the network behavior in CSSA, and design new dynamics for CR networks.

\section{TU Game Formulation AND Solution}

In this section, we model CSSA with payment transfer as a TU game. We prove that such a game has a nonempty core and propose a payoff sharing strategy for the game. Generally speaking, a TU game consists of a set of players and a function $v($.$) that associates with every nonempty subset of players a$ real number. The TU property implies that the total utility specified by this real number can be divided in any manner between the coalition members. The game is fully defined once the set of coalitions and the function $v($.$) are specified.$ The coalition values can be thought of as monetary values that the members in the coalition can distribute among themselves using an appropriate rule [17]. Thus, TU games have also been used to analyze market models. For example, [18] formulated a TU game for a market in which some units (e.g., houses, cars, etc.) are exchanged for money.

The analysis in this section also provides a market perspective: when the stable and optimal cooperative structure is formed, the payment each OP or SU obtains/pays associated with this structure will be determined. There are two kinds of players (OPs and SUs) in CSSA. A coalition including only OPs or SUs produce no valuable output. We define $v(\mathrm{~S})$ in CSSA as the maximum utility generated by the OPs and SUs in $\mathrm{S}$. Note that, the value of coalition $\mathrm{S}$ does not depend on the actions of the OPs or SUs in $N \cup \mathrm{K} \backslash \mathrm{S}$. The following convex optimization problem returns $v(\mathrm{~S})$ for any coalition $\mathrm{S} \subseteq \mathrm{N} \cup \mathrm{K}$ if feasible, else $v(\mathrm{~S})=-\infty$.

$\mathbf{P}(\mathrm{S})$ : Maximize: $\sum_{i \in \mathrm{S}_{o}}\left(U_{i}^{+}\left(y_{i}\right)-U_{i}^{-}\left(w_{i}\right)\right)+\sum_{k \in \mathrm{S}_{s}}\left(G_{k}^{+}\left(r_{k}\right)-\right.$ $\left.G_{k}^{-}\left(e_{k}\right)\right)$

\section{Subject to:}

(1) $y_{m}^{\prime}=\sum_{k \in \mathrm{S}_{s}} \beta_{m, k} I_{k, m}, m \in \mathrm{S}_{p}$.

(2) $w_{j}^{\prime}=\sum_{k \in \mathrm{S}_{s}} \alpha_{j, k}, j \in \mathrm{S}_{b}$.

(3) $r_{k}=\sum_{j \in \mathrm{S}_{b}} \alpha_{j, k} R_{j, k}, k \in \mathrm{S}_{s}$.

(4) $e_{k}=\sum_{m \in \mathrm{S}_{p}} \frac{1}{2} \beta_{m, k} P, k \in \mathrm{S}_{s}$.

(5) $\sum_{k \in \mathrm{S}_{s}} \alpha_{j, k} \leq 1, j \in \mathrm{S}_{b}$.

(6) $\sum_{k \in \mathrm{S}_{s}} \beta_{m, k} \leq 1, m \in \mathrm{S}_{p}$.

(7) $\sum_{m \in \mathrm{S}_{p}} \beta_{m, k}+\sum_{j \in \mathrm{S}_{b}} \alpha_{j, k} \leq 1, k \in \mathrm{S}_{s}$.

(8) $\alpha_{j, k}, \beta_{m, k}, y_{m}^{\prime}, w_{j}^{\prime}, r_{k}, e_{k} \geq 0, k \in \mathrm{S}_{s}, j \in \mathrm{S}_{b}, m \in \mathrm{S}_{p}$.

To make the resource allocation feasible in a coalition, in one unit of time, the total fraction of time that the SUs in coalition $\mathrm{S}$ gets to access a spare band is below 1, which is ensured by constraint (5). Constraint (6) means that the total fraction of time all SUs in the coalition uses to cooperate with a PU should be below 1. Constraint (7) guarantees that each SU works in relay or access mode, but not both at a given time. Constraint (8) is trivial. Any allocation $\left\{\beta_{m, k}, \alpha_{j, k}, m \in\right.$ $\left.\mathrm{S}_{p}, j \in \mathrm{S}_{b}, k \in \mathrm{S}_{s}\right\}$ that satisfies (5)-(8) is called a feasible joint action of coalition $\mathrm{S}$.

We now elucidate $v(S)$ and how cooperation can be formed between OPs and SUs using a simple example.

Example 1: Let $\mathrm{N}=\{a, b\}$, each $\mathrm{OP}$ has one $\mathrm{PU}$ and one spare channel. There are two SUs $K=\{1,2\}$ in the network. Let $j=1$ be the spare channel of OP $a$ and $j=2$ be the spare channel of OP $b$. Let $m=1$ represent the PU of OP $a$ and $m=$ 2 represent the PU of OP $b$. The transmission power $P=1$. The utility functions are set to be linear functions: $U_{i}^{+}\left(y_{i}\right)=$ $y_{i}, G_{k}^{+}\left(r_{k}\right)=r_{k}, U_{i}^{-}\left(w_{i}\right)=0.5 \times w_{i}$ and $G_{k}^{-}\left(e_{k}\right)=0.5 \times e_{k}$. 


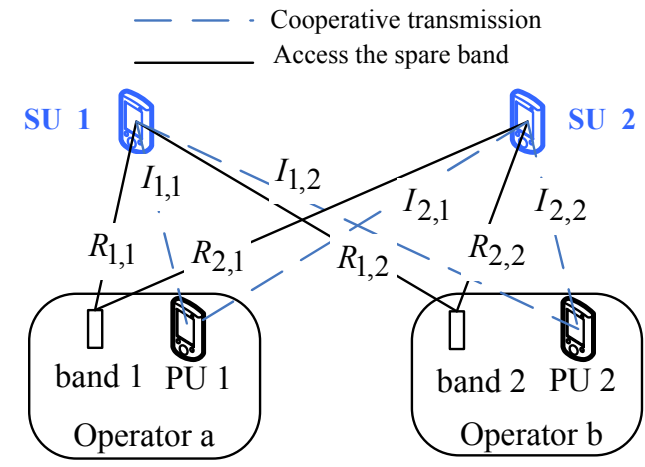

Fig. 3. An example to elucidate $v(\mathrm{~S})$ and how cooperation can be formed.

Assume that the access data rates of the SUs are $R_{1,1}=4$, $R_{1,2}=5, R_{2,1}=4, R_{2,2}=3$, and the throughput increases of the OPs are $I_{1,1}=3, I_{1,2}=2, I_{2,1}=6, I_{2,2}=5$ as shown in Fig. 3. Clearly, when OP $a$ or $b$ does not cooperate with SUs, it obtains a zero payoff. The same argument holds for SUs. Thus, only cooperative coalitions have the potential to generate positive payoffs. Consider a coalition $\{a, 1\}$, the optimal solution of $\mathbf{P}(\{a, 1\})$ which defines the coalition value results in an aggregate payoff of $R_{1,1}-0.5$. Assume that there is a payoff sharing strategy dividing $R_{1,1}-0.5$ into $\left(R_{1,1}-0.5\right) / 2$ for OP $a$ and SU 1 . In other words, the revenue OP $a$ obtained is $\left(R_{1,1}-0.5\right) / 2$.

Now, we consider the grand coalition $\{a, b, 1,2\}$. The optimal solution of $\mathbf{P}(\{a, b, 1,2\})$ assigns channel 2 to $\mathrm{SU}$ 1 , and assigns SU 2 to PU 1 as the relay. Then, the coalition value is $R_{1,2}-0.5+I_{2,1}-0.5$. This is socially desirable, since this coalition leads the maximum aggregate payoff in the system. However, if both OP $a$ and SU 1 receive a payoff less than $\left(R_{1,1}-0.5\right) / 2$, they will split from $\{a, b, 1,2\}$ and form $\{a, 1\}$. Thus, a payoff sharing strategy is required to ensure the stability of the socially desirable result.

Next, we introduce a solution concept for coalitional games known as core [16]. The idea behind the core of a cooperative game is analogous to that behind a Nash equilibrium of a noncooperative game: an outcome is stable if no deviation is profitable. That is, if a subset of OPs and SUs separates from the grand coalition then, irrespective of the manner in which the subset shares the aggregate payoff it generates after separating, at least one OP or SU is worse off. Formally, its definition is:

Definition 2: The core of CSSA TU game is the set of feasible payoff allocations:

$$
\begin{aligned}
\mathrm{C}= & \left\{\mathrm{x} \in R^{|\mathrm{N}|+|\mathrm{K}|}: \sum_{i \in \mathrm{N}} x_{i}+\sum_{k \in \mathrm{K}} x_{k}=v(\mathrm{~N} \cup \mathrm{K}),\right. \\
& \left.\sum_{i \in \mathrm{S}_{o}} x_{i}+\sum_{k \in \mathrm{S}_{s}} x_{k} \geq v(\mathrm{~S}), \forall \mathrm{S} \subset(\mathrm{N} \cup \mathrm{K})\right\} .
\end{aligned}
$$

This definition also means that there is no payoff vector $\mathbf{y}$ for a coalition $\mathrm{S} \subset \mathrm{N} \cup \mathrm{K}$ to make $y_{i}>x_{i}$ for all $i \in \mathrm{S}_{o}$, or $y_{k}>x_{k}$ for all $k \in \mathrm{S}_{s}$. Thus, no subgroup will separate from the grand coalition. To verify this, let OPs and SUs form the grand coalition and share the aggregate revenue $v(\mathrm{~N} \cup \mathrm{K})$ as per an imputation $\mathbf{x}, \mathbf{x} \in \mathrm{C}$. Suppose that a group of OPs and SUs (a coalition S) separates from the grand coalition and shares the aggregate payoff $v(\mathrm{~S})$ as per $\mathbf{z}$. Now, if $z_{i} \leq x_{i}$ or $z_{k} \leq x_{k}$ for $i \in \mathrm{S}_{o}$ and $k \in \mathrm{S}_{s}$, they have no incentive to separate from the grand coalition. Thus, $z_{i}>x_{i}, z_{k}>x_{k}$ for all $i \in \mathrm{S}_{o}, k \in \mathrm{S}_{s}$. Clearly, $\sum_{i \in \mathrm{S}_{o}} z_{i}+\sum_{k \in \mathrm{S}_{s}} z_{k}=v(\mathrm{~S})>$ $\sum_{i \in \mathrm{S}_{o}} x_{i}+\sum_{k \in \mathrm{S}_{s}} x_{k}$. This leads to a contradiction, since $\mathrm{x} \in \mathrm{C}$.

In the following, using the duality technique [19], we show that the game has a nonempty core, and get an element in it. For a coalition $\mathrm{S}$, let $\lambda, \pi \in R^{\left|\mathrm{S}_{b}\right|}, \mu, \delta \in R^{\left|\mathrm{S}_{p}\right|}, \eta, \gamma, \tau \in$ $R^{\left|\mathrm{S}_{s}\right|}$. Define:

$$
f_{i}(\delta, \pi)=\max _{\substack{y_{m}^{\prime} \geq 0, w_{j}^{\prime} \geq 0 \\ m \in \mathrm{M}_{i}, j \in \mathrm{B}_{i}}}\left(U_{i}^{+}\left(y_{i}\right)-U_{i}^{-}\left(w_{i}\right)+\sum_{m \in \mathrm{M}_{i}} \delta_{m} y_{m}+\right.
$$$$
\left.\sum_{j \in \mathrm{B}_{i}} \pi_{j} w_{j}\right) .
$$

$$
g_{k}(\gamma, \tau)=\max _{r_{k} \geq 0, e_{k} \geq 0}\left(G_{k}^{+}\left(r_{k}\right)-G_{k}^{-}\left(e_{k}\right)+\gamma_{k} r_{k}+\tau_{k} e_{k}\right) .
$$

Then, we have the following as a dual problem of $\mathbf{P}(\mathrm{S})$.

D(S): Minimize: $\sum_{i \in \mathrm{S}_{o}}\left(f_{i}(\delta, \pi)+\sum_{j \in \mathrm{B}_{i}} \lambda_{j}+\sum_{m \in \mathrm{M}_{i}} \mu_{m}\right)+$ $\sum_{k \in \mathrm{S}_{s}}\left(g_{k}(\gamma, \tau)+\eta_{k}\right)$.

\section{Subject to:}

1) $\pi_{j}+\gamma_{k} R_{j, k}+\eta_{k}+\lambda_{j} \geq 0, j \in \mathrm{S}_{b}, k \in \mathrm{S}_{s}$.

2) $\frac{1}{2} \tau_{k} P+\delta_{m} I_{m, k}+\eta_{k}+\mu_{m} \geq 0, k \in \mathrm{S}_{s}, m \in \mathrm{S}_{p}$.

3) $\tau_{k} \geq 0, \eta_{k} \geq 0, \gamma_{k} \geq 0, k \in \mathrm{S}_{s}$.

4) $\lambda_{j}, \pi_{j} \geq 0, j \in \mathrm{S}_{b}$. 5) $\delta_{m} \geq 0, \mu_{m} \geq 0, m \in \mathrm{S}_{p}$.

Formulate $\mathbf{D}(\mathrm{N} \cup \mathrm{K})$ by appropriately defining vectors $\lambda, \pi, \delta, \mu, \eta, \gamma$, and $\tau$. Denote $\mathrm{D}$ as the set of optimal solutions of $\mathbf{D}(\mathrm{N} \cup \mathrm{K})$. Then, define:

$$
\begin{aligned}
\mathrm{O}= & \left\{\mathrm{x}^{*} \in R^{|\mathrm{N}|+|\mathrm{K}|}:\right. \\
& x_{i}^{*}=f_{i}\left(\delta^{*}, \pi^{*}\right)+\sum_{j \in \mathrm{B}_{i}} \lambda_{j}^{*}+\sum_{m \in \mathrm{M}_{i}} \mu_{m}, i \in \mathrm{N} \\
& x_{k}^{*}=g_{k}\left(\gamma^{*}, \tau^{*}\right)+\eta_{k}^{*}, k \in \mathrm{K}, \\
& \text { for some } \left.\left(\delta^{*}, \pi^{*}, \lambda^{*}, \mu^{*}, \gamma^{*}, \tau^{*}, \eta^{*}\right) \in \mathrm{D}\right\} .
\end{aligned}
$$

Once $\mathrm{O}$ has been constructed, we have the following claim.

Theorem 1: The core of the coalitional game $\langle\mathrm{N} \cup \mathrm{K}, v\rangle$ with transferable utility is nonempty. And, $\mathrm{O} \subseteq \mathrm{C}$.

Proof: Clearly, $\mathrm{D} \neq \emptyset$. Thus, $\mathrm{O} \neq \emptyset$. We next show that for an arbitrary $\mathrm{x}^{*} \in \mathrm{O}, \mathrm{x}^{*} \in \mathrm{C}$. We consider an arbitrary $\mathbf{x}^{*} \in \mathrm{O}$ corresponding to $\left(\delta^{*}, \pi^{*}, \lambda^{*}, \mu^{*}, \gamma^{*}, \tau^{*}, \eta^{*}\right) \in \mathrm{D}$. Then, $\sum_{i \in \mathrm{N}} x_{i}^{*}+\sum_{k \in \mathrm{K}} x_{k}^{*}$ is the optimal value of the objective function of $\mathbf{D}(\mathrm{N} \cup \mathrm{K})$. Due to that $U_{i}^{+}($.$) and$ $G_{k}^{+}($.$) are concave functions, U_{i}^{-}($.$) and G_{k}^{-}($.$) are convex$ functions, thus the objective function of the problem $\mathbf{P}(\mathrm{S})$ is a concave function. Also, the constraints of $\mathbf{P}(\mathrm{S})$ are linear. Then, the dual gap is zero [19]. Thus, we can conclude that $\sum_{i \in \mathrm{N}} x_{i}^{*}+\sum_{k \in \mathrm{K}} x_{k}^{*}=v(\mathrm{~N} \cup \mathrm{K})$. According to the definition of the core, we now only need to show that $\sum_{i \in \mathrm{S}_{o}} x_{i}^{*}+\sum_{k \in \mathrm{S}_{s}} x_{k}^{*} \geq v(\mathrm{~S})$ for each $\mathrm{S} \subset \mathrm{N} \cup \mathrm{K}$.

By the strong duality, $v(\mathrm{~S})$ equals the optimal value of the objective function of $\mathbf{D}(\mathrm{S})$. The sub-vectors $\left(\delta_{S}, \pi_{S}, \lambda_{S}, \mu_{S}, \gamma_{S}, \tau_{S}, \eta_{S}\right)$ consisting of the components of $\left(\delta^{*}, \pi^{*}, \lambda^{*}, \mu^{*}, \gamma^{*}, \tau^{*}, \eta^{*}\right)$ in $\mathrm{S}$, satisfy the constraints of $\mathbf{D}(\mathrm{S})$. Then, $\sum_{i \in \mathrm{S}_{o}} x_{i}^{*}+\sum_{k \in \mathrm{S}_{s}} x_{k}^{*}$ is the value of the objective function of $\mathbf{D}(S)$ with such variables. The optimum value of 
the objective function of $\mathbf{D}(\mathrm{S})$ is a lower bound of $\sum_{i \in \mathrm{S}_{o}} x_{i}^{*}$ $+\sum_{k \in \mathrm{S}_{s}} x_{k}^{*}$. Thus, we can conclude that $\mathrm{x}^{*} \in \mathrm{C}$.

The above analysis provides the following insights. First, the solution of $\mathbf{P}(\mathrm{N} \cup \mathrm{K})$ defines the maximum cooperative profit of the system. Nevertheless, the utility evaluations of OPs or SUs directly derive from such a solution cannot ensure a stable and optimal coalition. Second, the set $\mathrm{O}$ determined by the dual solution of $\mathbf{P}(\mathrm{N} \cup \mathrm{K})$ constitutes a set of payoff vectors for the stable and optimal cooperative structure. Third, an OP/SU's payoff value specified by a payoff vector in $\mathrm{O}$ equals its utility evaluation reduced by the payment it is charged, or plus the revenue it obtains. Finally, the payment transfer in a coalition does not change the coalition value. Indeed, the analysis identified the pricing issue to motivate the optimal cooperation in the system and it was for the reason of payment transfer, one can get the stable and optimum solution of the TU game.

Shapley et al. [18] proposed two-sided assignment games and showed that the core of such games can be defined by the dual set of the linear optimization program. In [11], Aram et al. used the duality technique to solve a coalitional game which modeled the cooperation between base stations. Note that the structure of the optimization we considered is distinct from that of the above problems.

\section{Non-Transferable Payoff Coalition Game FORMULATION}

If the system does not support payment transfer, CSSA can be modeled as an NTU game in which each coalition $\mathrm{S}$ is characterized by a set $V(\mathrm{~S})$. Let $A(\mathrm{~S})$ denote the feasible joint action space of OPs and SUs in coalition $\mathrm{S}$ (all joint actions $\left\{\alpha_{j, k}^{S}, \beta_{m, k}^{S}, j \in \mathrm{S}_{b}, k \in \mathrm{S}_{s}, m \in \mathrm{S}_{p}\right\}$ satisfying the constraints of $\mathbf{P}(\mathrm{S})$ ). For a joint action $a^{S} \in A(\mathrm{~S})$, we define $F_{i}^{S}\left(a^{S}\right)=$ $U_{i}^{+}\left(y_{i}\right)-U_{i}^{-}\left(w_{i}\right)$ as the payoff value OP $i$ generates with $a^{S}$. Similarly, $F_{k}^{S}(a)=G_{k}^{+}\left(r_{k}\right)-G_{k}^{-}\left(e_{k}\right)$ is the payoff value SU $k$ generates with $a^{S}$. Let $F^{S}\left(a^{S}\right)$ be a payoff vector for all OPs and SUs in S. Then, $V(\mathrm{~S})$ is defined as:

$$
\begin{aligned}
V(\mathrm{~S})= & \left\{\mathbf{f} \in R^{\left|\mathrm{S}_{o}\right|+\left|\mathrm{S}_{s}\right|}: \exists \mathbf{z} \in R^{\left|\mathrm{S}_{o}\right|+\left|\mathrm{S}_{s}\right|}, \mathbf{z} \geq \mathbf{f},\right. \\
& \left.\mathbf{z}=F^{S}\left(a^{S}\right) \text { for some } a^{S} \in A(\mathrm{~S})\right\} .
\end{aligned}
$$

In other words, $V(\mathrm{~S})$ contains all payoff vectors that are less than or equal to at least one payoff vector generated by a feasible joint action. Any $\mathbf{f} \in V(\mathrm{~S})$ is called a feasible payoff vector. Note that, without utility transfer, payoff allocations in a coalition shall be exactly decided by the feasible joint actions. Next, the stage is set for the following definition for the solution of the game. The core of CSSA NTU game is:

$$
\begin{aligned}
\mathrm{C}_{N T U}= & \{\mathbf{f} \in V(\mathrm{~N} \cup \mathrm{K}): \forall \mathrm{S}, \nexists \mathbf{z} \in V(\mathrm{~S}) \text { such that } \\
& \left.z_{i}>f_{i} \text { or } z_{k}>f_{k}, \forall i \in \mathrm{S}_{o}, k \in \mathrm{S}_{s}\right\} .
\end{aligned}
$$

The payoff allocations in the core of the NTU game are also socially desirable, since the grand coalition has the potential to achieve higher efficiency. We now proceed to show that the core of CSSA NTU game $(\mathrm{N} \cup \mathrm{K}, V)$ is nonempty.

Definition 3: A collection of coalitions $I \subset 2^{\mathrm{N} \cup \mathrm{K}}$ is called balanced if there exist nonnegative weights $\lambda_{S}, \mathrm{~S} \in I$ such that:

$$
\sum_{\mathrm{S} \in I: i \in \mathrm{S}_{o}} \lambda_{S}=1, \forall i \in \mathrm{N}, \quad \sum_{\mathrm{S} \in I: k \in \mathrm{S}_{s}} \lambda_{S}=1, \forall k \in \mathrm{K},
$$

where $\mathrm{S} \in I: i \in \mathrm{S}_{o}$ means the coalitions in $I$, each containing OP $i$. The concept of balanced collection is quite obscure and it is useful to examine an example.

Example 2: Let $\mathrm{N}=\{a, b\}, \mathrm{K}=\{c, d\}$. Then, $I=$ $\{\{a, b, c\},\{b, d\},\{a, c, d\}\}$ is a balanced collection, since there exist balancing weights $\lambda_{S}=\frac{1}{2}, \forall \mathrm{S} \in I$ satisfying definition 3. Obviously, $I=\{\{a, b, c\},\{b, d\},\{c, d\}\}$ is not balanced, because there does not exist $\lambda_{S}, \forall \mathrm{S} \in I$ fulfilling definition 3 .

Definition 4: CSSA NTU game is balanced, if for every balanced collection $I$ a vector $\mathbf{f}=\left(f_{i}\right)_{i \in \mathrm{N}} \cup\left(f_{k}\right)_{k \in \mathrm{K}}$ must be in $V(\mathrm{~N} \cup \mathrm{K})$ if $\mathbf{f}^{S} \in V(\mathrm{~S})$ for all $\mathrm{S} \in I .{ }^{3}$

The general concept of a balanced game is due to Scarf [16]. We will make use of the following well known theorem which holds for any coalitional game.

Theorem 2 [16]: A balanced game always has a nonempty core.

Based on Theorem 2, we can obtain the following result.

Theorem 3: CSSA NTU game $(\mathrm{N} \cup \mathrm{K}, V)$ is balanced and hence has a nonempty core.

Proof: Consider a balanced collection $I$. Let $\left(\lambda_{S}, \mathrm{~S} \in\right.$ $I$ ) be the corresponding nonnegative weights of $I$. Let $\mathbf{f} \in$ $R^{|\mathrm{N}|+|\mathrm{K}|}$ be a vector with $\mathbf{f}^{S} \in V(\mathrm{~S})$ for each $\mathrm{S}$ in $I$. Then, there exists a joint action $\left\{\alpha_{j, k}^{S}, \beta_{m, k}^{S}, j \in \mathrm{S}_{b}, k \in \mathrm{S}_{s}, m \in \mathrm{S}_{p}\right\}$ for all $\mathrm{S} \in I$ such that:

1) $a^{S}=\left\{\alpha_{j, k}^{S}, \beta_{m, k}^{S}, j \in \mathrm{S}_{b}, k \in \mathrm{S}_{s}, m \in \mathrm{S}_{p}\right\} \in A(\mathrm{~S})$ satisfies the constraints of $\mathbf{P}(\mathrm{S}), \forall \mathrm{S} \in I$.

2) $f_{i} \leq U_{i}^{+}\left(y_{i}\right)-U_{i}^{-}\left(w_{i}\right), \forall i \in \mathrm{S}_{o}$, where $y_{i}$ and $w_{i}$ correspond to the joint action $a^{S} \cdot f_{k} \leq G_{k}^{+}\left(r_{k}\right)-G_{k}^{-}\left(e_{k}\right), \forall k \in \mathrm{S}_{s}$, where $r_{k}$ and $e_{k}$ are also determined by $a^{S}$.

Next, if we can show that the payoff vector $\mathbf{f} \in V(\mathrm{~N} \cup \mathrm{K})$, the game is balanced. Toward this end, we first need to define a joint action set $\left\{\alpha_{j, k}, \beta_{m, k}, j \in \mathrm{N}_{b}, k \in \mathrm{K}, m \in \mathrm{N}_{p}\right\}$ over the grand coalition such as $\alpha_{j, k}=\sum_{k \in \mathrm{S}_{s}, j \in I_{j}: \mathrm{S}_{b}} \lambda_{\mathrm{S}} \alpha_{k, j}^{S}$ where $\left\{\mathrm{S} \in I: k \in \mathrm{S}_{s}, j \in \mathrm{S}_{b}\right\}$ represents the coalitions $\mathrm{S}$ in $I$, each containing $k$ and $j$. Similarly, $\beta_{m, k}$ can be defined as $\beta_{m, k}=\sum_{k \in \mathrm{S}_{s}, m \in \mathrm{S}_{p}} \lambda_{\mathrm{S}} \beta_{m, k}^{S}$.

In what follows, the proof first proceeds by showing that $a^{\mathrm{N} \cup \mathrm{K}}=\left\{\alpha_{j, k}, \beta_{m, k}, j \in \mathrm{N}_{b}, k \in \mathrm{K}, m \in \mathrm{N}_{p}\right\} \in A(\mathrm{~N} \cup \mathrm{K})$. That is, $a^{\mathrm{N} \cup \mathrm{K}}$ is a feasible joint action over $\mathrm{N} \cup \mathrm{K}$. Subsequently, we prove that $F_{i}\left(a^{\mathrm{N} \cup \mathrm{K}}\right) \geq f_{i}, F_{k}\left(a^{\mathrm{N} \cup \mathrm{K}}\right) \geq f_{k}$, for $i \in \mathrm{N}, k \in \mathrm{K}$. Thus, according to equation (2), we can conclude that $\mathbf{f} \in V(\mathrm{~N} \cup \mathrm{K})$. The further proof consists of the following two steps:

Step 1: Prove that $a^{\mathrm{N} \cup \mathrm{K}}$ satisfies the feasible constraints

${ }^{3} \mathbf{f}^{S}$ is defined by $f_{i}^{S}=f_{i}, f_{k}^{S}=f_{k}, i \in \mathrm{S}_{o}, k \in \mathrm{S}_{s}$. A vector $\mathbf{f} \in$ $R^{|\mathrm{N}|+|\mathrm{K}|}$ with $\mathbf{f}^{S} \in V(\mathrm{~S})$ means that for each coalition there is a feasible joint action $a^{S} \in A(\mathrm{~S})$ to obtain the vector $\mathbf{f}^{S}$ such that $F_{i}^{S}\left(a^{S}\right) \geq f_{i}, i \in$ $\mathrm{S}_{o}, F_{k}^{S}\left(a^{S}\right) \geq f_{k}, k \in \mathrm{S}_{s}$. 
corresponding to the grand coalition.

$$
\begin{aligned}
& \sum_{m \in \mathrm{N}_{p}} \beta_{m, k}+\sum_{j \in \mathrm{N}_{b}} \alpha_{k, j} \\
= & \sum_{m \in \mathrm{N}_{p}} \sum_{\substack{\mathrm{S} \in I: \\
k \in \mathrm{S}_{s}, m \in \mathrm{S}_{p}}} \lambda_{S} \beta_{m, k}^{S}+\sum_{j \in \mathrm{N}_{b}} \sum_{\substack{\mathrm{S} \in I: \\
k \in \mathrm{S}_{s}, j \in \mathrm{S}_{b}}} \lambda_{S} \alpha_{j, k}^{S} \\
= & \sum_{\mathrm{S} \in I: k \in \mathrm{S}_{s}}\left(\lambda_{S} \sum_{m \in \mathrm{S}_{p}} \beta_{m, k}^{S}\right)+\sum_{\mathrm{S} \in I: k \in \mathrm{S}_{s}}\left(\lambda_{S} \sum_{j \in \mathrm{S}_{b}} \alpha_{k, j}^{S}\right) \\
= & \sum_{\mathrm{S} \in I: k \in \mathrm{S}_{s}} \lambda_{S}\left(\sum_{m \in \mathrm{S}_{p}} \beta_{m, k}^{S}+\sum_{j \in \mathrm{S}_{b}} \alpha_{k, j}^{S}\right) \\
\leq & \sum_{\mathrm{S} \in I: k \in \mathrm{S}_{s}} \lambda_{S}=1 .
\end{aligned}
$$

The first equality results from the definitions of $\beta_{m, k}$ and $\alpha_{k, j}$. The inequality follows by the feasibility of $a^{S}$ and constraint (7) of $\mathbf{P}(\mathrm{S})$. Similarly, we can show that constraints (5) and (6) of $\mathbf{P}(\mathrm{N} \cup \mathrm{K})$ are also fulfilled for $a^{\mathrm{N} \cup \mathrm{K}}$. Other constraints are trivial. Thus, the joint action $a^{\mathrm{N} \cup \mathrm{K}}$ is feasible.

Step 2: We show that $f_{i} \leq U_{i}^{+}\left(y_{i}^{\mathrm{N} \cup \mathrm{K}}\right)-U_{i}^{-}\left(w_{i}^{\mathrm{N} \cup \mathrm{K}}\right), \forall i \in$ $\mathrm{N}$, where $y_{i}^{\mathrm{N} \cup \mathrm{K}}$ denotes the throughput increase of OP $i$ and $w_{i}^{\mathrm{N} \cup \mathrm{K}}$ denotes the total secondary access time of OP $i$. Both of them correspond to the joint action $a^{\mathrm{N} \cup \mathrm{K}}$. Using the definition of $\beta_{m, k}$ and the fact that $\sum_{\mathrm{S} \in I: m \in \mathrm{S}_{p}} \lambda_{S}=1$, we can get that:

$$
\begin{aligned}
y_{i}^{\mathrm{N} \cup \mathrm{K}} & =\sum_{m \in \mathrm{M}_{i}}\left(\sum_{k \in \mathrm{K}} \beta_{m, k} I_{m, k}\right) \\
& =\sum_{m \in \mathrm{M}_{i}}\left(\sum_{k \in \mathrm{K}} \sum_{\substack{\mathrm{S} \in I: \\
k \in \mathrm{S}_{s}, m \in \mathrm{S}_{p}}} \lambda_{S} \beta_{m, k}^{S} I_{m, k}\right) \\
& =\sum_{m \in \mathrm{M}_{i}} \sum_{\mathrm{S} \in I: m \in \mathrm{S}_{p}} \lambda_{S}\left(\sum_{k \in \mathrm{S}_{s}} \beta_{m, k}^{S} I_{m, k}\right) \\
& =\sum_{m \in \mathrm{M}_{i}} \sum_{\mathrm{S} \in I: m \in \mathrm{S}_{p}} \lambda_{S} y_{m}=\sum_{\mathrm{S} \in I: i \in \mathrm{S}_{o}} \lambda_{S} y_{i} .
\end{aligned}
$$

That is to say that $y_{i}^{\mathrm{N} \cup \mathrm{K}}$ is the convex combination of $\left\{y_{i}, \mathrm{~S} \in I: i \in \mathrm{S}_{o}\right\}$. Similarly, it is easy to get that $w_{i}^{\mathrm{N} \cup \mathrm{K}}=\sum_{\mathrm{S} \in I: i \in \mathrm{S}_{o}} \lambda_{S} w_{i}$.

Since $U_{i}^{+}($.$) is concave and U_{i}^{-}($.$) is convex, for OP i$ we have:

$$
\begin{aligned}
U_{i}^{+}\left(y_{i}^{\mathrm{N} \cup \mathrm{K}}\right)-U_{i}^{-}\left(w_{i}^{\mathrm{N} \cup \mathrm{K}}\right) & \geq \sum_{\mathrm{S} \in I: i \in \mathrm{S}_{o}} \lambda_{S}\left(U_{i}^{+}\left(y_{i}\right)-U_{i}^{-}\left(w_{i}\right)\right) \\
& =\sum_{\mathrm{S} \in I: i \in \mathrm{S}_{o}} \lambda_{S} F_{i}^{S}\left(a_{S}\right)=f_{i} .
\end{aligned}
$$

Following the same argument, we can also show that $f_{k} \leq$ $G_{k}^{+}\left(z_{k}\right)-G_{k}^{-}\left(e_{k}\right), \forall k \in \mathrm{K}$. Then, based on steps 1,2 and Theorem 2, the core of the game is nonempty.

A resource allocation game has been studied where OPs can serve each other's subscribers to achieve their own best payoff [11]. Following the basic concept of balanced games, the authors showed that the resulting game has a nonempty core. Also, a similar idea has been used to investigate the core solutions of coalitional games in economics. It is worth noting that the core stability and the balanced conditions are very restrictive. In many coalitional games, there are no core solutions. It is usually interesting and meaningful to reveal that the new sensible coalitional game models are balanced.
As far as CSSA NTU game is concerned, the properties we considered, i.e., the cooperative diversity transmissions and two kinds of game players, make our formulation distinct from previous research work in this area.

\section{Computation of a Member in the Core of NTU GAME}

To implement CSSA, another important issue is to calculate an element in the core. Scarf [16] first studied the core of a balanced game. The main result of his work is to derive the core existence resulting from a constructive algorithm over balanced games. Note that to compute a payoff profile in the core using this method, we first have to construct a finite list of payoff profiles whose size is exponential in parameters of the network. As a result, the implementation is centralized and the computational time will be exponential. The interested readers can refer to [16] [12] for details about such a general method. In this section, we mainly focus on constructing efficient algorithms for the special situations where the access costs of OPs and the cooperative costs of SUs are negligible or zero. That is $U_{i}^{-}(.) \approx 0, \forall i \in \mathrm{N}$ and $G_{k}^{-}(.) \approx 0, \forall k \in \mathrm{K}$.

Under the special situations mentioned above, we can relate CSSA NTU game to an exchange economy setting, a concept borrowed from microeconomics. Then, we prove that the competitive equilibrium in the exchange economy setting, if exists, corresponds to a payoff profile in the core of CSSA NTU game. Finally, we show that the equilibrium exists, which can be computed using several available efficient centralized or distributed algorithms.

\section{A. Exchange economy preliminaries}

We first introduce the preliminaries about exchange economy. An exchange economy consists of: (1) a set of agents $\mathcal{A}$ (the set of agents), (2) a positive integer $\ell$ representing the number of goods, (3) for each agent $l \in \mathcal{A}$, an endowment vector $q_{l} \in R_{+}^{\ell}$ such that every component of $\sum_{l \in \mathcal{A}} q_{l}$ is positive, and (4) for each agent $l \in \mathcal{A}$, a nondecreasing, continuous and quasi-concave utility function ${ }^{4} u_{l}():. R_{+}^{\ell} \rightarrow R$ over the set $R_{+}^{\ell}$ of bundles of goods.

Goods transfer between the agents, but there is no payoff that is freely transferable. $q_{l}$ is the bundle of goods that agent $l$ owns initially. The requirement that every component of $\sum_{l \in \mathcal{A}} q_{l}$ is positive means that there is a positive quantity of every good available in the economy. An allocation is a distribution of the total endowment in the economy among the agents: a profile $\left(x_{l}\right)_{l \in \mathcal{A}}$ with $x_{l} \in R_{+}^{\ell}$ for all $l \in \mathcal{A}$ and $\sum_{l \in \mathcal{A}} x_{l}=\sum_{l \in \mathcal{A}} q_{l}$. In other words, it is possible to provide agents with a desired allocation just using the total endowment of the economy.

Let a vector $\mathbf{p}=\left(p_{1}, \ldots, p_{\ell}\right)$ denote the prices of the goods in the market ${ }^{5}$. The agents will then try to maximize their own utility through goods' exchanges according to the prices given by $\mathbf{p}$. A competitive equilibrium of an exchange economy is

\footnotetext{
${ }^{4} \mathrm{~A}$ function $u(x)$ mapping from the reals to the reals is quasi-concave if for any two points in the domain, say $x_{1}$ and $x_{2}$, the value of $u(x)$ on all points between them satisfies: $u(x)>=\min \left\{u\left(x_{1}\right), u\left(x_{2}\right)\right\}$.

${ }^{5}$ The price vector does not engage in the exchange economy, it is just employed to analyze the equilibrium property of an exchange economy.
} 


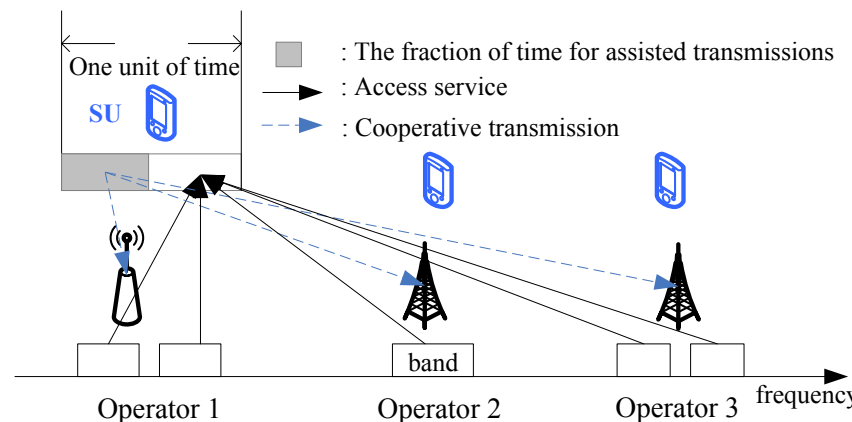

Fig. 4. Exchange economy setting for CSSA.

a pair $\left(\mathbf{p}^{*},\left(x_{l}^{*}\right)_{l \in \mathcal{A}}\right)$ consisting of a vector $\mathbf{p}^{*} \in R_{+}^{\ell}$ with $\mathbf{p}^{*} \neq 0$ (the price vector), and an allocation $\left(x_{l}^{*}\right)_{l \in \mathcal{A}}$ such that for each agent $l$ we have:

$x_{l}^{*} \in \arg \max _{x_{l} \in R_{+}^{\ell}} u_{l}\left(x_{l}\right)$ subject to $\mathbf{p}^{*} \cdot x_{l} \leq \mathbf{p}^{*} \cdot q_{l}, \forall l \in \mathcal{A}$.

If $\left(\mathbf{p}^{*},\left(x_{l}^{*}\right)_{l \in \mathcal{A}}\right)$ is a competitive equilibrium, then $\left(x_{l}^{*}\right)_{l \in \mathcal{A}}$ is called a competitive allocation. Note that $\mathbf{p}^{*} \cdot x_{l}$ is the value of agent $l$ 's allocation after exchanging which clearly cannot be larger than the value of her endowment (budget constraint). The equilibrium condition guarantees that the total supply of every good equals the total demand of it and hence the market clears. The pioneering equilibrium theorem of Arrow and Debreu [20] showed that:

Theorem 4 [20]: Every exchange economy defined above has a Competitive Equilibrium (CE) with the property that the price vector is strictly positive.

\section{B. CSSA NTU game and exchange economy}

Consider CSSA NTU game defined in section VI. Now, think of OPs and SUs in N $\cup \mathrm{K}$ to be agents in the exchange economy. Then, there are two kinds of goods in the economy. The one is the spare channels owned by OPs given in one unit of time and the other is the cooperative transmissions of SUs given in one unit of time as well. From the point of each OP, there are $|\mathrm{K}|$ kinds of choices for cooperative transmissions, since different SUs/relays engender different data rate gains. OPs and SUs trade their initial endowments to get what they need from each other. An example of the exchange economy setting for CSSA is drawn in Fig. 4.

Recall that the set $\mathrm{N}_{b}$ consists of all spare bands. We array all spare bands and SUs to form a set $\mathrm{T}=\mathrm{N}_{b} \cup \mathrm{K}$ with the cardinality of $\left|\mathrm{N}_{b}\right|+|\mathrm{K}|$. Now, let $j^{\prime}$ represent the rank number of band $j$ in $\mathrm{N}_{b}$, and let $k^{\prime}$ represent the rank number of SU $k$ in K. Denote $t$ as the rank number of an SU in T. Hence, the SU represented by $t=\left|\mathrm{N}_{b}\right|+k^{\prime}$ refers to the same $\mathrm{SU}$ as $k, k \in \mathrm{K}$. In the rest of the paper, unless otherwise specified, $t$ always equals $\left|\mathrm{N}_{b}\right|+k^{\prime}$. Then, we can construct an initial resource vector $\mathbf{q}\left(\mathbf{q} \in R_{+}^{\ell}, \ell=\left|\mathrm{N}_{b}\right|+|\mathrm{K}|\right)$ for OPs and SUs as follows:

- For OP $i, i \in \mathrm{N}$, let $q_{i}^{j^{\prime}}=1$ be the fraction of time OP $i$ holds for band $j$ and $q_{i}^{j^{\prime}}=0$, otherwise.

- Similarly, for $\mathrm{SU} k, k \in \mathrm{K}$, let $q_{k}^{j^{\prime}}=0$, since $\mathrm{SU}$ cannot access the spare channels initially. Let $q_{k}^{t}=1$ be the fraction of time held by SU $k$ (includes idle state, cooperative transmission time and accessing time) and $q_{k}^{t}=0, t \neq\left|\mathrm{N}_{b}\right|+k^{\prime}$, otherwise.

We consider an allocation $\mathbf{x}=\left(x_{i}^{*}\right)_{i \in \mathrm{N}} \cup\left(x_{k}^{*}\right)_{k \in \mathrm{K}}$ of the economy setting with an initial resource vector $\mathbf{q}$ defined above. Then, $x_{i} \in R_{+}^{\ell}$ and $x_{k} \in R_{+}^{\ell}$ determines the allocated resource for each OP and each SU, respectively. In particular, $x_{i}^{t}$ is the fraction of time $\mathrm{SU} k$ uses to assist primary transmissions of OP $i . x_{k}^{j^{\prime}}$ represents the fraction of time that SU $k$ obtains to access channel $j . x_{k}^{t}$ means the total faction of time SU $k$ has for accessing or being idle.

Then, SUs' utilities can be defined as the maximum payoffs they can obtain through accessing the spare bands, which are specified by allocation $\mathrm{x}$ :

$$
u_{k}\left(x_{k}\right)=\max _{\alpha_{j, k}, j \in \mathrm{N}_{b}} G_{k}^{+}\left(r_{k}\right), \quad k \in \mathrm{K} .
$$

\section{Subject to:}

1) $\left.r_{k}=\sum_{j \in \mathrm{N}_{b}} \alpha_{j, k} R_{j, k} .2\right) \alpha_{j, k} \leq x_{k}^{j^{\prime}}, j \in \mathrm{N}_{b}$.

3) $\sum_{j \in \mathrm{N}_{b}} \alpha_{j, k} \leq x_{k}^{t}$. 4) $\alpha_{j, k} \geq 0, j \in \mathrm{N}_{b}$.

Clearly, $u_{k}($.$) is a nondecreasing, continuous and quasi-$ concave function. In order to employ the available efficient algorithms which require the concave property of the utility function, for some cases, we can apply the monotone transformation from $u_{k}($.$) to a concave function that is "equivalent"$ to such a utility function.

Proposition 1: If $G_{k}^{+}($.$) is a linear function, i.e., G_{k}^{+}\left(r_{k}\right)=$ $c_{k} \times r_{k}$ where $c_{k}$ is a utility factor, then $\log \left(u_{k}().\right)$ is a concave function.

Proof: First, according to the value of $R_{j, k}$, we array $\left\{R_{j, k}, j \in \mathrm{N}_{b}\right\}$ in descending order. Let $n \in\left\{1, \ldots,\left|\mathrm{N}_{b}\right|\right\}$ denote a rank number which specifies a spare band exactly. Then, let $R_{n, k}^{\prime}$ denote the access rate of SU $k$ in descending order. Denote $\mathrm{x}_{k}^{n}$ as the fraction of time that SU $k$ obtains to access channel $n$. Note that, the value of $\mathbf{x}_{k}^{n}$ is determined by $x_{k}$. Hence, $u_{k}\left(x_{k}\right)$ can be rewritten as:

$$
\begin{aligned}
& u_{k}\left(x_{k}\right)=\min \left\{x_{k}^{t}, \mathbf{x}_{k}^{1}\right\} \times c_{k} R_{1, k}^{\prime} \\
& +\min \left\{\max \left\{0,\left(x_{k}^{t}-\mathbf{x}_{k}^{1}\right)\right\}, \mathbf{x}_{k}^{2}\right\} \times c_{k} R_{2, k}^{\prime}+\ldots \\
& +\min \left\{\max \left\{0,\left(x_{k}^{t}-\ldots-\mathbf{x}_{k}^{\left|\mathrm{N}_{b}\right|-1}\right)\right\}, \mathbf{x}_{k}^{\left|\mathrm{N}_{b}\right|}\right\} \times c_{k} R_{\left|\mathrm{N}_{b}\right|, k}^{\prime} .
\end{aligned}
$$

That is, with the constraint of time $x_{k}^{t}$, SU $k$ always attempts to allocate the access time to its favorite spare band.

Clearly, we can get that $\forall \alpha>0, u_{k}\left(\alpha x_{k}\right)=\alpha u_{k}\left(x_{k}\right)$ which means that $u_{k}\left(x_{k}\right)$ is a homogeneous function ${ }^{6}$ with degree one. We now apply Friedman's theorem [21] which states that a monotone, homogeneous and quasi-concave function is log concave. Hence, $\log \left(u_{k}().\right)$ is concave.

For each OP, the utility function is defined to be the maximum payoff it can obtain from the cooperative transmissions for its PUs, which is specified by allocation $\mathrm{x}$ as:

$$
u_{i}\left(x_{i}\right)=\max _{\substack{\beta_{m, k}, k \in \mathrm{K}, m \in \mathrm{M}_{i}}} U_{i}^{+}\left(y_{i}\right), \quad \forall i \in \mathrm{N} .
$$

\section{Subject to:}

1) $y_{m}=\sum_{k \in \mathrm{K}} \beta_{m, k} I_{m, k}, m \in \mathrm{M}_{i}$.

2) $\sum_{k \in \mathrm{K}} \beta_{m, k} \leq 1, m \in \mathrm{M}_{i}$.

3) $\sum_{m \in \mathrm{M}_{i}} \beta_{m, k} \leq x_{i}^{t}, k \in \mathrm{K}$.

${ }^{6} \mathrm{~A}$ function $u($.$) is homogeneous of degree \mathrm{d}$ if for any $\alpha>0, u(\alpha x)=$ $\alpha^{d} u(x)$. 
4) $\beta_{m, k} \geq 0, k \in \mathrm{K}, m \in \mathrm{M}_{i}$.

For any feasible allocation $x_{i}$, constraint (2) in the optimization defining $u_{i}($.$) is never binding. If U_{k}^{+}($.$) is a$ linear function, i.e., $U_{k}^{+}\left(r_{k}\right)=c_{k} \times r_{k}$. Then, $u_{i}\left(x_{i}\right)$ can be represented as $u_{i}\left(x_{i}\right)=\max \left\{c \cdot \beta \mid \beta \in R_{+}^{\left|\mathrm{M}_{i}\right| \times|\mathrm{K}|}, A \beta \leq x_{i}\right\}$ where $c \in R_{+}^{\left|\mathrm{M}_{i}\right| \times|\mathrm{K}|}$ and the columns of matrix $A$ can be thought of as "objects" that the trader wants to "build". Such a function is also a homogeneous function with degree one [25]. Then, $\log \left(u_{i}\left(x_{i}\right)\right)$ is also a concave function.

Remark: In this paper, we mainly study the linear cases for $U_{k}^{+}($.$) and G_{k}^{+}($.$) . However, under situations where u_{k}($.$) and$ $u_{i}($.$) are quasi-concave, the results presented in this section$ always hold.

Each of the self-interested OP or SU in the exchange economy attempts to get an allocation benefiting itself as much as possible. Similar to the concept of core in coalitional games, one can use the core of the exchange economy as a policy to determine the resource allocation for OPs and SUs. Formally, its definition is given as :

$$
\mathrm{C}^{e}=\left\{\mathrm{x}^{*} \in R_{+}^{(|\mathrm{N}|+|\mathrm{K}|) \ell}: \sum_{h \in \mathrm{N} \cup \mathrm{K}} x_{h}^{*} \leq \sum_{h \in \mathrm{N} \cup \mathrm{K}} q_{h}, \forall \mathrm{S},\right.
$$

$\nexists \mathbf{z} \in R_{+}^{(|\mathrm{N}|+|\mathrm{K}|) \ell}$, such that $u_{h}\left(z_{h}\right)>u_{h}\left(x_{h}^{*}\right), h \in \mathrm{S}_{o} \cup \mathrm{S}_{s}$, and $\left.\sum_{h \in \mathrm{S}_{o} \cup \mathrm{S}_{s}} z_{h} \leq \sum_{h \in \mathrm{S}_{o} \cup \mathrm{S}_{s}} q_{h} \cdot\right\}$,

where $\ell=\left|\mathrm{N}_{b}\right|+|\mathrm{K}|$. That is, an allocation $\mathbf{x}^{*}=\left(x_{i}^{*}\right)_{i \in \mathrm{N}} \cup$ $\left(x_{k}^{*}\right)_{k \in \mathrm{K}}$ is in the core of the exchange economy, if it cannot be blocked by any coalition $\mathrm{S}, \mathrm{S} \subset \mathrm{N} \cup \mathrm{K}$. There is a general property about the core of an exchange economy.

Theorem 5 [17]: Every competitive allocation of an exchange economy is in its core.

We will show that an allocation in the core of an exchange economy setting corresponds to a feasible joint action in the grand coalition, and a payoff profile in the core of CSSA NTU game. Consequently, using Theorem 5, the available efficient algorithms to compute the cooperative allocation of an exchange economy can be used to get a profile in the core of CSSA NTU game. Toward this end, we first present Lemma 1.

Lemma 1: For any payoff vector $\hat{\mathbf{z}} \in V(\mathrm{~S})$, there exists an allocation $\hat{\mathrm{x}}$ of the economy setting such that:

a) $\sum_{h \in \mathrm{S}_{o} \cup \mathrm{S}_{s}} \hat{x}_{h} \leq \sum_{h \in \mathrm{S}_{o} \cup \mathrm{S}_{s}} q_{h}$.

b) $u_{h}\left(\hat{x}_{h}\right) \geq \hat{z}_{h}, \forall h \in \mathrm{S}_{o} \cup \mathrm{S}_{s}$.

Proof: Recall that, $V(\mathrm{~S})$ contains all payoff vectors that are less than or equal to at least one payoff vector generated by a feasible joint action. Suppose that a joint action $\left\{\beta_{m, k}, \alpha_{j, k}, m \in \mathrm{S}_{p}, j \in \mathrm{S}_{b}, k \in \mathrm{S}_{k}\right\}$ corresponds to $\hat{\mathbf{z}}$. Define $\hat{x}_{i}^{t}=\sum_{m \in \mathrm{M}_{i}} \beta_{m, k}, k \in \mathrm{S}_{s}, i \in \mathrm{S}_{o}$. Define $\hat{x}_{k}^{j^{\prime}}=\alpha_{j, k}, j \in \mathrm{S}_{b}, k \in \mathrm{S}_{s}$, and $\hat{x}_{i}^{j^{\prime}}=0, i \in \mathrm{S}_{o}$. That is, the amount of channel $j$ owned by OP $i$ is set to zero. Since $\left\{\beta_{m, k}, \alpha_{j, k}, m \in \mathrm{S}_{p}, j \in \mathrm{S}_{b}, k \in \mathrm{S}_{s}\right\}$ is a feasible joint action of $\mathrm{S}$, it satisfies the constraints of $\mathbf{P}(\mathrm{S})$. Then, we get:

$$
\begin{aligned}
& \sum_{h \in \mathrm{S}_{o} \cup \mathrm{S}_{s}} \hat{x}_{h}^{t}=\sum_{i \in \mathrm{S}_{o}} \hat{x}_{i}^{t}+\sum_{k \in \mathrm{S}_{s}} \hat{x}_{k}^{t}=\sum_{i \in \mathrm{S}_{o}} \sum_{m \in \mathrm{M}_{i}} \beta_{m, k}+\sum_{k \in \mathrm{S}_{s}} \alpha_{j, k} \\
& =\sum_{m \in \mathrm{S}_{p}} \beta_{m, k}+\sum_{k \in \mathrm{S}_{s}} \alpha_{j, k} \leq 1=\sum_{h \in \mathrm{S}_{o} \cup \mathrm{S}_{s}} q_{h}^{t}, k \in \mathrm{S}_{s},
\end{aligned}
$$

where the last equality comes from the definition of $q_{i}$ and $q_{k}$. In the same way, one can easily verify that $\sum_{h \in \mathrm{S}_{o} \cup \mathrm{S}_{s}} \hat{x}_{h}^{j^{\prime}} \leq$ $\sum_{h \in \mathrm{S}_{\circ} \cup \mathrm{S}_{s}} q_{h}^{j^{\prime}}, j \in \mathrm{S}_{b}$.

Next, note that, for the given $\hat{x}_{i},\left\{\beta_{m, k}, k \in \mathrm{S}_{s}, m \in \mathrm{S}_{p}\right\}$ constitutes a feasible solution of the optimization defining $u_{i}\left(\hat{x}_{i}\right)$, and $\hat{z}_{i}$ is the value of the objective function. Thus, $u_{i}\left(\hat{x}_{i}\right) \geq \hat{z}_{i}, \forall i \in \mathrm{S}_{o}$. A similar argument holds for $u_{k}\left(\hat{x}_{k}\right) \geq \hat{z}_{k}, k \in \mathrm{S}_{s}$. Then, b) immediately follows.

For any allocation $\mathrm{x}^{*}$ belonging to $\mathrm{C}^{e}$, assume that the joint action $\left\{\beta_{m, k}^{*}, \alpha_{j, k}^{*}, m \in \mathrm{N}_{p}, j \in \mathrm{N}_{b}, k \in \mathrm{K}\right\}$ consists of optimal solutions of the optimizations defining $u_{i}\left(x_{i}^{*}\right), i \in \mathrm{N}_{o}$ and $u_{k}\left(x_{k}^{*}\right), k \in \mathrm{K}$. Then, based on Lemma 1, we get the following theorem:

Theorem 6: If $\mathbf{z}$ is the payoff vector that corresponds to $\left\{\beta_{m, k}^{*}, \alpha_{j, k}^{*}, m \in \mathrm{N}_{p}, j \in \mathrm{N}_{b}, k \in \mathrm{K}\right\}$, then $\mathbf{z} \in \mathrm{C}_{N T U}$.

Proof: Suppose that $\mathbf{z} \notin \mathrm{C}_{N T U}$, then there exists a coalition $\mathrm{S}$ and a payoff profile $\hat{\mathbf{z}} \in V(\mathrm{~S})$ such that $\hat{z}_{i}>z_{i}, \forall i \in \mathrm{S}_{o}$ or $\hat{z}_{k}>z_{k}, \forall k \in \mathrm{S}_{s}$. Since $\hat{z} \in V(\mathrm{~S})$, by Lemma 1 , there exists an allocation $\hat{\mathbf{x}}$ such that: a) $\sum_{h \in \mathrm{S}_{o} \cup \mathrm{S}_{s}} \hat{x}_{h} \leq \sum_{h \in \mathrm{S}_{o} \cup \mathrm{S}_{s}} q_{h}$, b) $u_{i}\left(\hat{x}_{i}\right) \geq \hat{z}_{i}, \forall i \in$ $\mathrm{S}_{o}$ or $u_{k}\left(\hat{x}_{k}\right) \geq \hat{z}_{k}, k \in \mathrm{S}_{s}$. Consequently, $u_{i}\left(\hat{x}_{i}\right) \geq \hat{z}_{i}>$ $z_{i}, \forall i \in \mathrm{S}_{o}$ or $u_{k}\left(\hat{x}_{k}\right) \geq \hat{z}_{k}>z_{k}, k \in \mathrm{S}_{s}$. This is in contradiction with $\mathrm{x}^{*} \in \mathrm{C}^{e}$.

Using Theorem 6, we argue that:

Corollary 1: If $\left(\mathbf{p}^{*}, \mathbf{x}^{*}\right)$ is a CE of the exchange economy setting, then the corresponding payoff vector $u_{i}\left(x_{i}^{*}\right)_{i \in \mathrm{N}} \cup$ $u_{k}\left(x_{k}^{*}\right)_{k \in \mathrm{K}}$ is in the core of CSSA NTU game.

To illustrate the concept of $\mathrm{CE}$, we consider a price vector p. OP $i$ or SU $k$ chooses a bundle which is the most desirable among all those that are affordable (i.e., satisfy $\mathbf{p} \cdot x_{i} \leq \mathbf{p} \cdot q_{i}$ ). Typically, for a price vector $\mathbf{p}$, the demand vector of OP $i$ can be defined as $d_{i}(\mathbf{p})=\max _{x_{i} \in R_{+}^{e}} u_{i}\left(x_{i}\right)$ subject to $\mathbf{p} \cdot x_{i} \leq$ $\mathbf{p} \cdot q_{i}$. The demand vector of SU $k$ is defined as $d_{k}(\mathbf{p})=$ $\max u_{k}\left(x_{k}\right)$ subject to $\mathbf{p} \cdot x_{k} \leq \mathbf{p} \cdot q_{k}$. That is, the competitive $x_{k} \in R_{+}^{\ell}$

allocation is an allocation of resource for OP $i$ or $\mathrm{SU} k$, which maximizes the utility and subjects to the budget constraint.

Moreover, the aggregate excess demand in the exchange economy is a function $\xi$ given by $\xi(\mathbf{p})=\sum_{i \in \mathrm{N}}\left(d_{i}(\mathbf{p})-q_{i}\right)+$ $\sum_{k \in \mathrm{K}}\left(d_{k}(\mathbf{p})-q_{k}\right)$, i.e., the aggregate demand minus the total endowment. $\mathbf{p}^{*}$ is an equilibrium price vector, if $\xi\left(\mathbf{p}^{*}\right)=0$. In other words, if agents trade goods at the prices of $\mathbf{p}^{*}$, then for each good the sum of the individuals' demand equals the sum of their supplies. The above claim has pointed out that a payoff profile in the core of the NTU game can be obtained by computing the corresponding $\mathrm{CE}$.

\section{Computation of the competitive equilibrium}

Fruitful results in computational economics provided us a wide range of methods to compute the CE. Smale developed a global Newton's method for the computation of equilibrium prices [22]. The solver in [23] improved Smale's algorithm by doing a line search within each Newton's iteration. Such methods, despite not being guaranteed to run in polynomial time, they have demonstrated the capability of handling real world applications [23]. The decentralized price adjustment process [24] required that utility functions are concave to 


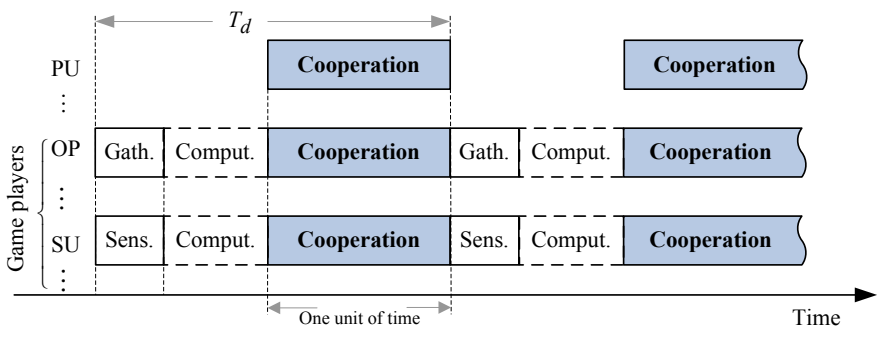

Fig. 5. Implementation of CSSA. Gath., Sens. and Comput. mean Gathering, Sensing and Computing, respectively.

get a CE. Recent works [25], [26] presented fast distributed algorithms under certain conditions.

Some researchers analyzed the practical performance of different algorithms. In [28], the efficiency of Newton's method is investigated. The performance of interior point methods has been analyzed in [27]. The authors surveyed algorithms to compute $\mathrm{CE}$ and indicated how these algorithms can be improved from the computational point of view.

Having the CE allocation, based on theorem 6, it is easy to get the resulting payoff vector $u_{i}\left(x_{i}^{*}\right)_{i \in \mathrm{N}} \cup u_{k}\left(x_{k}^{*}\right)_{k \in \mathrm{K}}$ to be in the core of CSSA NTU game. The focus of this paper is not the design of algorithms. In Section VIII, we just employ the algorithm in [25] for an example to illustrate the core allocation of CSSA NTU game.

\section{IMPLEMENTATION OF CSSA}

In this section, the implementation of CSSA in practical networks is discussed. Specifically, we illustrate how CSSA can be realized with and without a payment mechanism.

A rough sketch of the construction of CSSA is shown in Fig. 9 from which we can find a unified description of the implementations of CSSA for two situations. Firstly, we take the TU model as an example. one CSSA course is divided into multiple slots with length $T_{d}$ (as shown in Fig. 5), each corresponding to one round in CSSA. All network parameters (including the number of channels, the number and locations of SUs, the number of PUs, the utility evaluations of SUs and OPs, etc.) are assumed to be constant in one CSSA duration $\left(T_{d}\right)$. Suppose that there is a central computer with the complete information of network parameters and a common control channel used for the interaction of signals. The central auctioneer has also been applied to some spectrum auction models, e.g., [4], [6].

In the initialization of each round, each OP broadcasts a flag packet over all spare bands to inform SUs. This means that SUs can obtain channel conditions of all spare bands. Then, SUs are scheduled to send a packet (serveral bits enough) over the pilot channels. When PUs acquire the channel conditions from SUs, each of them piggybacks such information to its access point ${ }^{7}$. Then, the channel information will be gathered by OPs. According to the above issues, a whole CSSA duration can be designed with the following three phases:

1) Sensing and gathering, in which SUs sense the spare bands and OPs gather the channel information.

\footnotetext{
${ }^{7}$ In practical networks, the central controller can restrict the number of SUs and PUs involved in CSSA to save the exchanged messages.
}

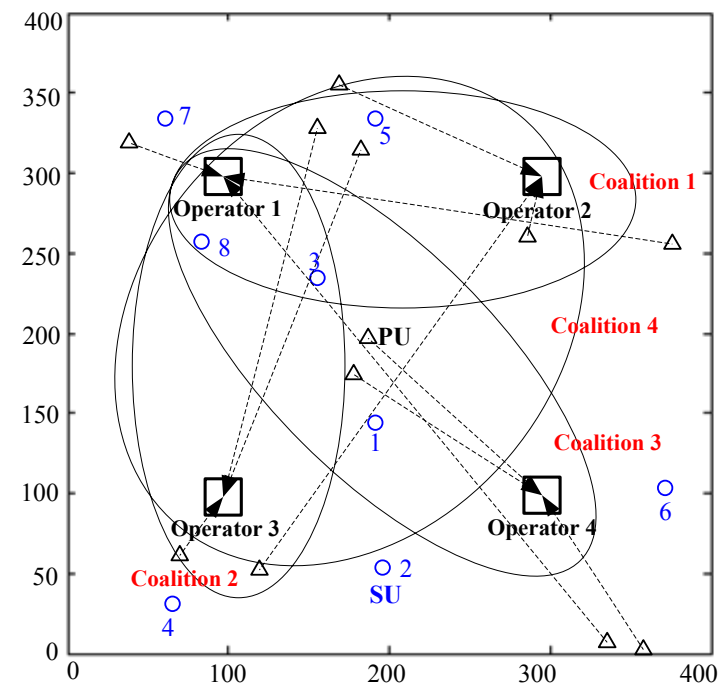

Fig. 6. Network model.

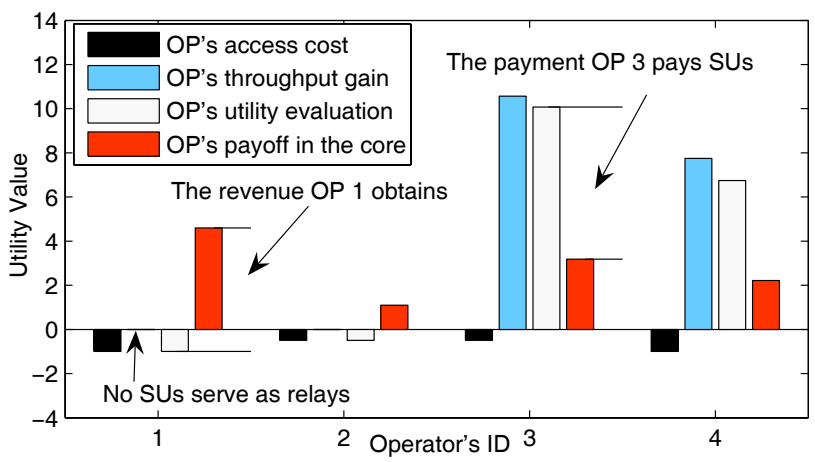

(a) The utility evaluations of SUs and their payoffs in the core.

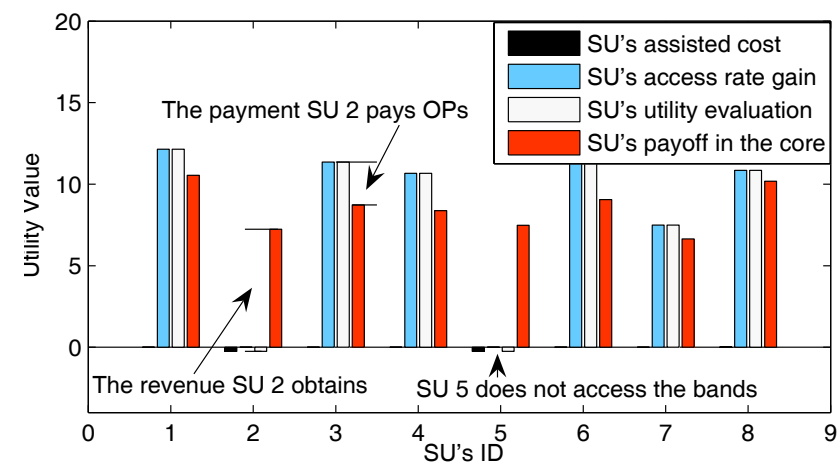

(b) The utility evaluations of OPs and their payoffs in the core.

Fig. 7. The performance of CSSA and the solution of the TU game.

2) Computing, the central computer is in charge of the payment, as well as determines the action of each SU or OP by the method in Theorem 1.

3) Cooperation, in which SUs access bands and perform cooperative transmissions for PUs.

Furthermore, if some SUs and OPs leave the grand coalition and form a new coalition, no matter what payment they make and what actions they take, it is impossible to provide a better allocation for all members in the new coalition.

Now, we turn to the NTU model. The implementation of CSSA is constructed as before, but there is no central 

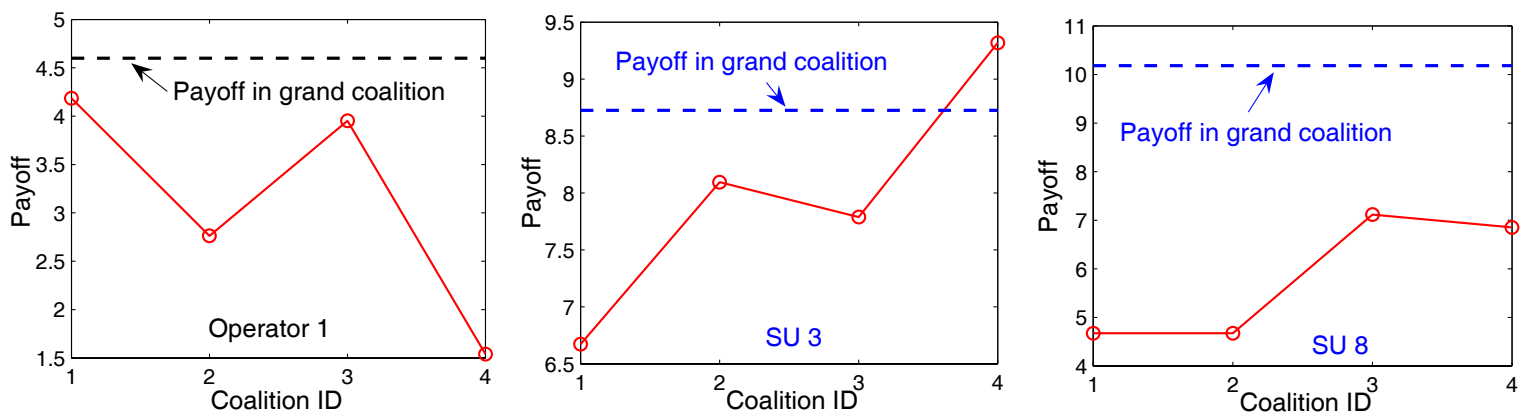

Fig. 8. The stability of grand coalition.

controller to support payment management. Additionally, alternative techniques can be used to compute an element in the core. For instance, the calculation can be performed by the centralized algorithms [22] [23] [27]. Or, it is done through distributed interactions [24] [25] [26] between all SUs and OPs to achieve the CE.

\section{NUMERICAL RESULTS}

In this section, we present numerical results to illustrate the cooperative actions of OPs and SUs in CSSA. Also, we unveil different solutions between the TU model and the NTU model by an example. Firstly, we study the case with transferable utility. We set 4 OPs in the network on a square area of $400 \mathrm{~m} \times$ $400 \mathrm{~m}$ as shown in Fig. 6. The numbers of spare bands of the OPs are set to 3,2,2 and 3, respectively. Assume that each OP has 3 PUs. All PUs and SUs are randomly placed in the area. The transmission power of each node is set to 1 . We assume that the noise variances at receivers are the same and set to $\delta^{2}=10^{-6}$. The channel gain between any two nodes is modeled as an i.i.d. zero-mean complex circular Gaussian random variable with $C N(0,1)$ distribution.

Let the utility functions be linear functions such as $U_{i}^{+}\left(y_{i}\right)=5 \times y_{i}$ and $G_{k}^{+}\left(r_{k}\right)=g_{a} \times r_{k}$ where $g_{a}$ is an access rate gain factor. Set $U_{i}^{-}\left(w_{i}\right)=0.5 \times w_{i}$ and $G_{k}^{-}\left(e_{k}\right)=0.5 \times e_{k}$. As we know, the coalition value $v(S)$ which is defined by the convex optimization problem $\mathbf{P}(\mathrm{S})$, quantifies the worth of a coalition in the game. When all SUs and OPs form the grand coalition, the solution of $\mathbf{P}(\mathrm{N} \cup \mathrm{K})$ determines the throughput gain of each $\mathrm{OP}$, the access rate gain of each SU, the access cost of each OP and the energy cost of each SU. We plot these results in Fig. 7. Nevertheless, the utility evaluations cannot guarantee a stable grand coalition.

The core of the game is directly related to the grand coalition's stability. The solution of the dual problem $\mathbf{D}(\mathrm{N} \cup \mathrm{K})$ constitutes a payoff allocation in the core of the TU game. Specifically, such an aggregate payoff sharing strategy determines the payment each OP/SU obtains or pays. In Fig. 7, we set $g_{a}=10$ and plot such a payoff allocation placing with the utility evaluations. From the figure, we clearly observe that payment transfers from some agents to others. For instance, SU 5 does not access spare bands and OP 1 does not get throughput gain, but they receive extra revenue.

The payoff allocation in the core guarantees that no group of players has an incentive to leave the grand coalition to form another coalition. This property is studied in Fig. 8.

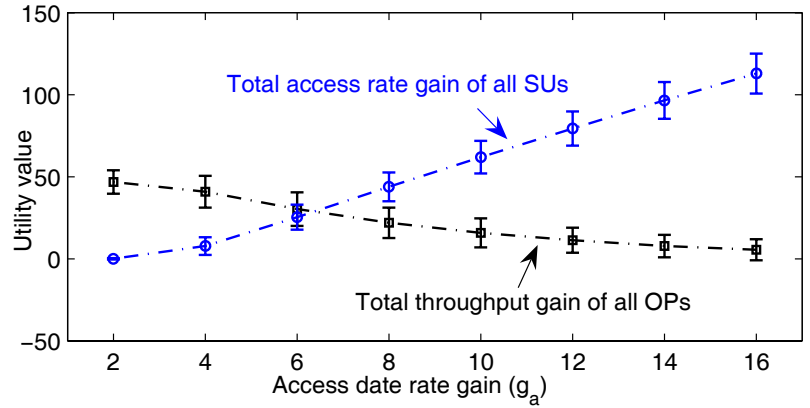

(a) Cooperation gains.

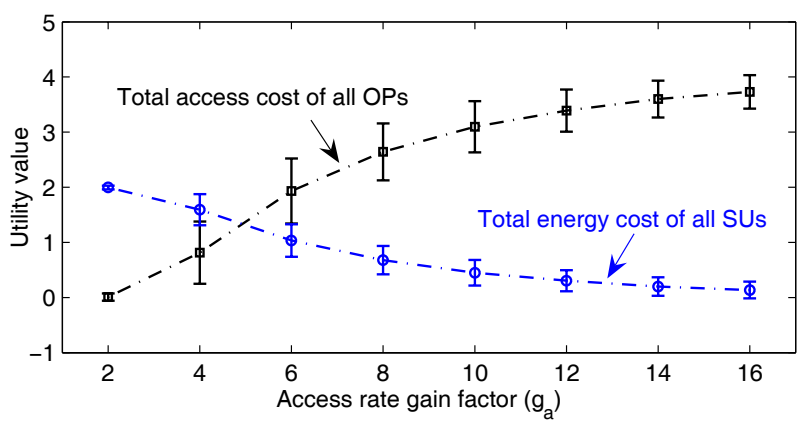

(b) Cooperation costs.

Fig. 9. The impact of access rate gain factor $\left(g_{a}\right)$ on the cooperation scheme.

We construct some sub coalitions in the network as shown in Fig. 6. Some agents such as OP 1, SU 3 and SU 8 are contained in all sub coalitions. Fig. 8 illustrates that leaving the grand coalition does not provide higher payoffs for all of them.

Fig. 9 shows the impact of access rate gain factor $\left(g_{a}\right)$ on the cooperation scheme. Fig. 9(a) reveals that, with increasing $g_{a}$, the total throughput gain of all OPs decreases. The reason is that a larger value of $g_{a}$ makes OPs get less fraction of time of cooperative transmissions to achieve the optimal coalition value. However, SUs obtain more fraction of time for access. Hence, the total access cost of all OPs rises and the total energy cost of all SUs reduces. We illustrate this in Fig. 9(b). The total payment each side (all OPs or all SUs) generates with different $g_{a}$ is plotted in Fig. 10, from which we find that the payment one side receives exactly equals to the payment another side consumes. This means that the payment transfer in the coalition does not change the coalition value.

Next, we use a simple example to study the model without 


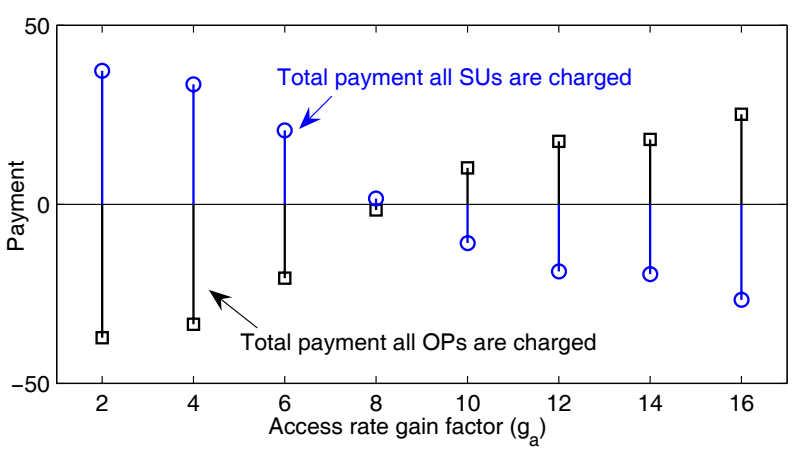

Fig. 10. The impact of access rate gain factor $\left(g_{a}\right)$ on the payment transfer.

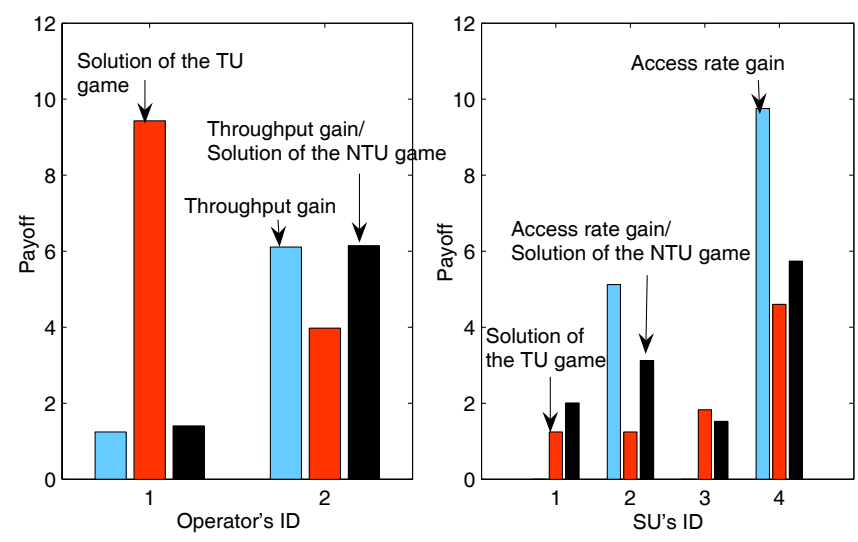

Fig. 11. The solutions of the TU game and the NTU game.

any transferable payoff. We set two OPs and assume that each OP has one PU and one spare band. There are four SUs in the network. For the case where the access costs of OPs and the cooperative costs of SUs are zero or negligible, we set $U_{i}^{-}()=0,. G_{k}^{-}()=$.0 . Then, a simple exchange economy setting is constituted. Fig. 11 illustrates the different results between the TU and NTU games. They clearly show that, in the NTU game, we do not get a stable and socially optimal solution. This is due to the fact that there is no charge being introduced into the payoff allocation of the NTU game to support the stable and socially optimal solution. However, without payment management, the deployment of commercial networks will be much more convenient.

\section{CONCLUSION}

In this paper, we have developed new dynamics for the cooperative CR networks with multiple OPs and SUs. First, we considered the scenario in which a payment mechanism was supported by the system. Such a situation led to a TU coalitional game. Based on the dual theory, the cooperative strategies and the payment each OP or SU was charged for the stable and optimal solution of the game have been determined. Secondly, we investigated an interesting scenario where there is no payment transfer in the CR network. We modeled the system by an NTU game and proved that the core of such a game is nonempty. Under special situations, we related an exchange economy setting to the NTU game. As a result, some available efficient centralized or distributed algorithms to compute $\mathrm{CE}$ can be employed to get a solution of the game. We discussed in detail the implementation of new dynamics in practical networks. Numerical studies have been performed to reveal the rational cooperative actions of OPs and SUs in CSSA for two situations. In summary, this paper has introduced techniques by which coalitional behavior of cognitive radios can be analyzed, thereby enabling the efficient design of coalition based CR dynamics for future CR networks.

\section{ACKNOWLEDGMENT}

This work is supported by National Fundamental research grant (2011CB302701, 2010CB731803, 2009CB3020402), NSF China (No. 60702046, 60832005, 60972050, 60632040); China Ministry of Education (No. 20070248095); China Ministry of Education Fok Ying Tung Fund (No. 122002); Qualcomm Research Grant; China International Science and Technology Cooperation Programm (No. 2008DFA11630); PUJIANG Talents (08PJ14067); Shanghai Innovation Key Project (08511500400); National Key Project of China (2009ZX03003-006-03, 2009ZX03002-003, 2009ZX03002005,2010ZX03003-001-01); National High tech grant of China (2009AA01Z248, 2009AA1Z249, 2009AA011802).

\section{REFERENCES}

[1] S. Force, "Spectrum policy task force report," Federal Communications Commission ET docket 02, vol. 135, 2002.

[2] O. Simeone, I. Stanojev, S. Savazzi, Y. Bar-Ness, U. Spagnolini, and R. Pickholtz, "Spectrum leasing to cooperating secondary ad hoc networks," IEEE J. Sel. Areas Commun., vol. 26, no. 1, pp. 203-213, Jan. 2008.

[3] J. Zhang and Q. Zhang, "Stackelberg game for utility-based cooperative cognitive radio networks," in Proc. ACM MobiHoc 2009, New Orleans, USA, May 2009.

[4] J. Huang, R. A. Berry, and M. L. Honig, "Auction-based spectrum sharing," ACM Mobile Networks and Applications, vol. 11, no. 3, pp. 405-418, June 2006.

[5] D. Niyato and E. Hossain, "Market-equilibrium, competitive, and cooperative pricing for spectrum sharing in cognitive radio networks: analysis and comparison," IEEE Trans. Wireless Commun., vol. 7, no. 11 , pp. 4273-4283, Nov. 2008.

[6] G. S. Kasbekar and S. Sarkar, "Spectrum auction framework for access allocation in cognitive radio networks" in Proc. ACM MobiHoc 2009, New Orleans, USA, May 2009.

[7] D. Niyato, E. Hossain, and Z. Han, "Dynamics of multiple-seller and multiple-buyer spectrum trading in cognitive radio networks: a game theoretic modeling approach," IEEE Trans. Mobile Comput., vol. 8, no. 8, pp. 1009-1022, Aug. 2009.

[8] W. Saad, Z. Han, M. Debbah, A. Hjøungnes, and T. Başar, "Coalitional game theory for communication networks," IEEE Signal Process. Mag., vol. 26, no. 5, pp. 77-97, Sep. 2009.

[9] Z. Han and H. Vincent Poor, "Coalition games with cooperative transmission: a cure for the curse of boundary nodes in selfish packetforwarding wireless networks," IEEE Trans. Commun., vol. 57, no. 1, pp. 203-213, Jan. 2009.

[10] S. Mathur, L. Sankar, and N. B. Mandayam, "Coalitions in cooperative wireless networks," IEEE J. Sel. Areas Commun., vol. 26, no. 7, pp. 1104-1115. Sep. 2008.

[11] A. Aram, C. Singh, S. Sarkar, and A. Kumar, "Cooperative profit sharing in coalition based resource allocation in wireless networks," in Proc. IEEE INFOCOM 2009, Rio de Janeiro, Brazil, Apr. 2009.

[12] A. Aram, C. Singh, S. Sarkar, and A. Kumar, "Provider and customer game," in Proc. IEEE GameNet 2009, Istanbul, Turkey, May 2009.

[13] K. Azarian, H. E. Gamal, and P. Schniter, "On the achievable diversitymultiplexing tradeoff in half-duplex cooperative channels," IEEE Trans. Inf. Theory, vol. 51, no. 12, pp. 4152-4172, Dec. 2005.

[14] A. Chowdhery and R. K. Mallik, "Linear dection for the nonnorthogonal amplify and forward protocol," IEEE Trans. Wireless Commun., vol. 8, no. 2 , pp. 826-835, Feb. 2009. 
[15] R. U. Nabar, H. Bölcskei, and F. W. Kneubühler, "Fading relay channels: performance limits and space-time signal design," IEEE J. Sel. Areas Commun., vol. 22, no. 6, Aug. 2004.

[16] H. E. Scarf, "The core of an n person game," Econometrica, vol. 35, no. 1 pp. 50-69, Jan. 1967.

[17] M. Osborne and A. Rubinstein, A Course in Game Theory. MIT Press, 1999.

[18] L. Shapley and M. Shubik, "The assignment game I: the core," Int'l J. Game Theory, vol. 1, no. 1, pp. 111-130, 1972.

[19] S. Boyd and L. Vandenberghe, Convex Optimization. Cambridge University Press, 2004.

[20] K. J. Arrow and G. Debreu, "Existence of an equilibrium for a competitive economy," Econometrica, vol. 22, no. 3, pp. 265-290, July 1954.

[21] K. Jain, V. V. Vazirani, and Y. Ye, "Market equilibria for homothetic, quasi-concave utilities and economies of scale in production," in Proc. ACM Symposium on Discrete Algorithms, Vancouver, British Columbia, Jan. 2005.

[22] S. Smale, "A convergent process of price adjustment and global newton methods," J. Mathematical Economics, vol. 3, pp. 107-120, July 1976.

[23] M. C. Ferris and T. S. Munson, "Complementarity problems in games and the path solver," J. Economic Dynamics and Control, vol. 24, no. 2, pp. 165-188, Feb. 2000.

[24] R. Joosten and D. Talman, "A globally convergent price adjustment process for exchange economies," J. Mathematical Economics, vol. 29, no. 1, pp. 15-26, Jan. 1998.

[25] L. Fleischer, R. Garg, S. Kapoor, R. Khandekar, and A. Saberi, "A fast and simple algorithm for computing market equilibria," in Proc. International Workshop on Internet and Network Economics, Shanghai, China, Dec. 2008.

[26] B. Codenotti, B. McCune, and K. Varadarajan, "Market equilibrium via the excess demand function," in Proc. ACM Symposium on Theory of Computing, Baltimore, MD, May 2005.

[27] M. Esteban-Bravom, "Computing equilibria in general equilibrium models via interior-point methods," Computational Economic, vol. 23, no. 2 pp. 147-171, 2004.

[28] P. Harker and B. Xiao, "Newton's method for the nonlinear complementarity problem: a b-differential equation approach," Mathematical Programming, vol. 48, no. 1 pp. 339-357, 1990.

[29] H. Yu, L. Gao, Z. Li. X. Wang, and E. Hossain, "Pricing for uplink power control in cognitive radio networks," IEEE Trans. Veh. Technol., vol. 59, no. 4, pp. 1769-1778, 2010.

[30] X. Wang, Z. Li, P. Xu, Y. Xu, X. Gao, and H. Chen, "Spectrum sharing in cognitive radio networks-an auction based approach," IEEE Trans. System, Man and Cybernetics-Part B: Cybernetics, vol. 40, no. 3, pp. 587-596, 2010.

[31] Mo $\mathrm{Li}$ and Yunhao Liu, "Underground coal mine monitoring with wireless sensor networks," ACM Trans. Sensor Networks, vol. 5, no. 2, Mar. 2009.

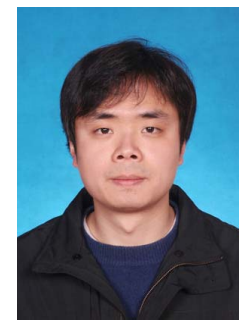

Dapeng Li received the B.S. degree in Electronics from Harbin Engineering University, China, in 2003, and the M.S. degree in Communication Systems from Harbin Engineering University, China, in 2006. He received his Ph.D. degree from the Department of Electronic Engineering, Shanghai Jiao Tong University, China, in 2010. Currently, he is a faculty member in the College of Telecommunications and Information Engineering, Nanjing University of Posts and Telecommunications, Nanjing, China. His research interests include future cellular networks, mobile ad hoc networks, cognitive radio networks, radio resource management and cooperative communications.

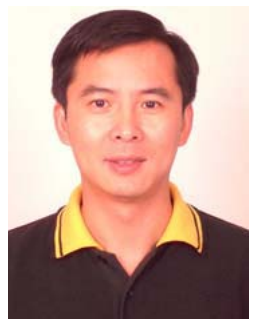

Youyun Xu was born in 1966. He was graduated from Shanghai Jiao Tong University with Ph.D. Degree in Information and Communication Engineering in 1999. He is currently a professor with Nanjing Institute of Communication Engineering, China. $\mathrm{He}$ is also a part-time professor with the Institute of Wireless Communication Technology of Shanghai Jiao Tong University (SJTU), China. Dr. $\mathrm{Xu}$ has more than 20 years professional experience of teaching and researching in communication theory and engineering. Now, his research interests are focusing on New Generation Wireless Mobile Communication System (IMT-Advanced and Related), Advanced Channel Coding and Modulation Techniques, Multi-user Information Theory and Radio Resource Management, Wireless Sensor Networks, Cognitive Radio Networks, etc. He is a member of IEEE, a senior member of Chinese Institute of Electronics.

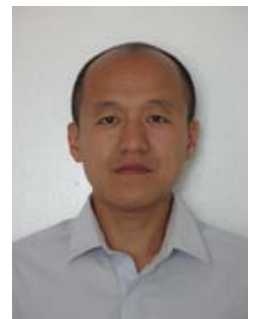

Xinbing Wang received the B.S. degree (with hons.) from the Department of Automation, Shanghai Jiaotong University, Shanghai, China, in 1998, and the M.S. degree from the Department of Computer Science and Technology, Tsinghua University, Beijing, China, in 2001. He received the Ph.D. degree, major in the Department of electrical and Computer Engineering, minor in the Department of Mathematics, North Carolina State University, Raleigh, in 2006. Currently, he is a faculty member in the Department of Electronic Engineering, Shanghai Jiaotong University, Shanghai, China. His research interests include resource allocation and management in mobile and wireless networks, TCP asymptotics analysis, wireless capacity, cross layer call admission control, asymptotics analysis of hybrid systems, and congestion control over wireless ad hoc and sensor networks. Dr. Wang has been a member of the Technical Program Committees of several conferences including IEEE INFOCOM 2009-2011, IEEE ICC 2007-2011, IEEE Globecom 2007-2011.

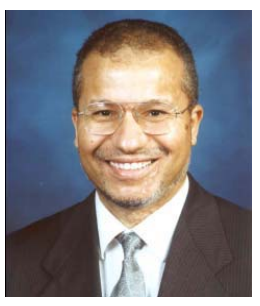

Mohsen Guizani is currently a Professor and the Associate Dean of Academic Affairs at Kuwait University. He was the Chair of the CS Department at Western Michigan University from 2002 to 2006 and Chair of the CS Department at University of West Florida from 1999 to 2002. He also served in academic positions at the University of MissouriKansas City, University of Colorado-Denver and Syracuse University. He received his B.S. (with distinction) and M.S. degrees in Electrical Engineering; M.S. and Ph.D. degrees in Computer Engineering in 1984, 1986, 1987, and 1990, respectively, from Syracuse University, Syracuse, New York. His research interests include Computer Networks, Wireless Communications and Mobile Computing, and Optical Networking. He currently serves on the editorial boards of six technical Journals and the Founder and EIC of the journal Wireless Communications and Mobile Computing, published by John Wiley (http://www.interscience.wiley.com/jpages/15308669/), and the Journal of Computer Systems, Networks and Communications (http://www.hindawi.com/journals/), published by Hindawi. He is also the Founder and the Steering Committee Chair of the Annual International Conference of Wireless Communications and Mobile Computing (IWCMC). $\mathrm{He}$ is the author of seven books and more than 200 publications in refereed journals and conferences. He guest edited a number of special issues in IEEE journals and magazines. He also served as member, Chair, and General Chair of a number of conferences. Dr. Guizani is currently the Chair of WTC and was Chair of TAOS ComSoc Technical Committees. He was an IEEE Computer Society Distinguished Lecturer from 2003 to 2005. Dr. Guizani is an IEEE Fellow and a Senior member of ACM. 